\title{
Practical considerations for using K3 cameras in CDS mode for high- resolution and high-throughput single particle cryo-EM
}

\author{
Ming Sun ${ }^{*}$, , Caleigh Azumaya*,a, Eric Tse ${ }^{*, b}$, Adam Frost ${ }^{\text {a,c }}$, Daniel Southworth ${ }^{b}$, Kliment A. Verba ${ }^{\mathrm{c}, \mathrm{d}}$, Yifan Cheng ${ }^{\mathrm{a}, \mathrm{e}}$ and \\ David A. Agard ${ }^{\text {a }}$,** \\ ${ }^{\text {a }}$ Department of Biochemistry \& Biophysics, University of California, San Francisco, San Francisco, CA, 94143, United States \\ ${ }^{\mathrm{b}}$ Institute for Neurodegenerative Diseases, University of California, San Francisco, San Francisco, CA, 94143, United States \\ ${ }^{c}$ Quantitative Biosciences Institute (QBI), University of California, San Francisco, San Francisco, CA, 94143, United States \\ ${ }^{\mathrm{d}}$ Department of Pharmaceutical Chemistry, University of California, San Francisco, San Francisco, CA, 94143, United States \\ ${ }^{\mathrm{e}}$ Howard Hughes Medical Institute, University of California, San Francisco, San Francisco, CA, 94143, United States \\ * Authors contributed equally. \\ *** Correspondence: agard@ @msg.ucsf.edu, University of California, San Francisco, San Francisco, CA, 94143, United States
}

\begin{abstract}
Detector technology plays a pivotal role in high-resolution and high-throughput cryo-EM structure determination. Compared with the first-generation, single-electron counting direct detection camera (Gatan K2), the latest K3 camera is faster, larger, and now offers a correlated-double sampling mode (CDS). Importantly this results in a higher DQE and improved throughput compared to its predecessor. In this study, we focused on optimizing camera data collection parameters for daily use within a cryo-EM facility and explored the balance between throughput and resolution. In total, eight data sets of murine heavy-chain apoferritin were collected at different dose rates and magnifications, using 9-hole image shift data collection strategies. The performance of the camera was characterized by the quality of the resultant 3D reconstructions. Our results demonstrated that the Gatan K3 operating in CDS mode outperformed nonCDS mode in terms of reconstruction resolution in all tested conditions with 8 electrons per pixel per second being the optimal dose rate. At low magnification (64kx) we were able to achieve reconstruction resolutions of $149 \%$ of the physical Nyquist limit (1.8 $\AA$ with a $1.346 \AA$ physical pixel). Low magnification allows more particles to be collected per image, aiding analysis of heterogeneous samples requiring large data sets. At moderate magnification $(105 \mathrm{kx}, 0.834 \AA$ physical pixel size) we achieved a resolution of $1.65 \AA$ within 9 hours of data collection, a condition optimal for achieving high-resolution on well behaved samples. Our results also show that for an optimal sample like apoferritin, one can achieve better than $2.5 \AA$ resolution with 5 minutes of data collection. Together, our studies validate the most efficient ways of imaging protein complexes using the $\mathrm{K} 3$ direct detector and will greatly benefit the cryo-EM community.
\end{abstract}

Keywords: Cryo-EM; direct detector; high-end data collection

\section{Introduction}

Cryo-electron microscopy (cryo-EM), particularly single particle cryo-EM, has experienced tremendous success in its ability to provide structural information on how dynamic molecules perform their biological functions. Through technological advances in both hardware and software, it is now possible to determine structures of a wide range of biological complexes at or near atomic resolution using cryo-EM. Recently, the achievable resolution of single particle cryo-EM has been extended to well beyond $2 \AA$, where individual atoms can be accurately localized in the cryo-EM density map (Nakane et al., 2020; Yip et al., 2020; Zhang et al., 2020). However, achieving such high resolution comes at a cost of time and resources, which will influence how broadly the technology will be employed to address fundamental biological questions.

Detector technology plays a pivotal role in high-resolution and high-throughput structure determination and drove the 'resolution revolution' in cryo-EM. The current direct detectors combine direct detection and single electron counting, which allows dramatic improvements in the Detective Quantum Efficiency (DQE) well beyond that previously possible. DQE is one of the most important quantitative metrics characterizing the performance of EM cameras and varies as a function of spatial frequency (McMullan et al., 2009, 2016; Li, Mooney et al., 2013; Mooney, 2007). Higher DQE values indicate less noise added by the detector itself to the final images. Therefore, maximizing DQE across all frequencies is a major objective during the detector design. During the course of detecting single electron events, the incident electron can distribute charge in adjacent pixels, allowing the incident location to be estimated to higher precision (known as super-resolution mode on Gatan cameras) (Li, Zheng et al., 2013; Booth et al., 2012). The $2 \mathrm{x}$ increased sampling reduces aliasing artifacts and provides the ability for usable information to go beyond the physical Nyquist. 
Sun et al., 08 Nov 2020 - preprint copy - BioRxiv

The Gatan K3 detector (Ametek Gatan) further improves DQE compared to the previous generation K2 Summit detector by having improved image processing, a $4 \mathrm{x}$ higher internal frame rate and a higher pixel count. While many factors affect DQE in any single electron counting camera, for a given pixel design and sensitive layer thickness, two important ones are analog noise and frame rate. The former determines the smallest signal from an incoming electron that can be reliably counted, while the latter dictates the degree of systematic undercounting of events due to two electrons arriving within the frame readout interval (known as coincidence loss) ((Li, Zheng et al., 2013); Figure S1). The effects of coincidence loss can be visualized in noise power spectrum (NPS) curves (Figure S2; Table S1 and S2). For any given dose rate, the higher the internal camera frame rate the less coincidence loss and the higher the effective DQE. A practical trade-off is that choosing to work at lower dose rates translates into longer exposures and reduced throughput. Compared with the K2, the K3 also successfully implements a correlated double sampling (CDS) imaging mode which suppresses analog noise (McMullan et al., 2016) allowing a more reliable detection of weak events. This is accomplished by effectively subtracting a dark frame from each frame recorded, taking two read cycles. Thus, there is a dose-rate dependent trade-off between better detection in CDS mode vs. reduced coincidence loss in nonCDS mode.

To optimize the usage of the K3 for daily operation, we explored the balance between throughput and resolution and characterized the performance of the camera by evaluating the quality of 3D reconstructions from data collected at different dose rates and magnifications. Magnification determines the image pixel size (both physical and super-resolution) and Nyquist resolution limits. Since the DQE of a camera is a function of Nyquist resolution, altering the magnification places different spatial frequencies in different DQE ranges of the camera. At a given spatial resolution, higher magnification directly translates into improved DQE but comes at the expense of a reduced effective imaging area, limiting the number of particles that can be recorded within each image.

Apoferritin, a standard sample for evaluating data quality in cryo-EM ( $\sim 500 \mathrm{kDa}$ with octahedral symmetry) was collected at $300 \mathrm{kV}$ on our standard X-FEG Titan Krios equipped with a Gatan BioQuantum energy filter and $\mathrm{K} 3$ camera. To maximize throughput, we utilized image shift (Mastronarde, 2005; Suloway et al., 2005) rather than stage motion to image nearby holes. In our collection scheme, a central point is localized using stage shift and images are shot in a regular $3 \times 3$ pattern of holes around the central location using a combination of beam tilt and image shift. At $64 \mathrm{kx}$ we are able to reach $1.8 \AA$, $50 \%$ higher than the $2.69 \AA$ physical Nyquist frequency from 373,000 selected particles within 9 hours of image-shift data collection. This reconstruction far surpasses the physical Nyquist resolution in a standard data collection period in our facility. At $105 \mathrm{kx}$, we reached $1.65 \AA$, $101 \%$ of the $1.67 \AA$ physical Nyquist frequency, from 571,000 selected particles within 8 hours of data collection. In as short as 5 minutes, from 7,000 particles, apoferritin can be reconstructed to under $2.5 \AA$ resolution using a $1.34 \AA$ physical pixel size, under ideal conditions. In our higher magnification maps, we were able to discern high-resolution features including densities for hydrogens in the peptide backbone and certain amino acid side chains. Our results demonstrate that the Gatan K3 operating in CDS mode outperforms nonCDS mode in terms of reconstruction resolution in all tested conditions with 8 electrons per pixel per second (eps) being the most efficient dose rate to reach high-resolution.

\section{$\underline{\text { Results }}$}

\section{Imaging benchmark cryo-EM samples under different conditions on the Gatan K3 camera}

In this study, we imaged apoferritin to systematically characterize the performance of the K3 camera with and without using CDS mode, as well as to elucidate the relationship between two important practical aspects of single particle cryo-EM: attainable resolution vs. throughput. Summarized in Table 1, we collected a total of eight datasets of apoferritin at different dose rates and magnifications. As described in detail in the methods section, all data sets were collected using the identical, whenever possible, or comparable microscope settings. The three most important variables we explored were magnification, dose rate, and CDS mode. Different dose rates were achieved by adjusting spot size without changing additional microscope parameters. Exposure times were adjusted so that the total electron dose per each image stack was held constant. Additionally, physical pixel sizes are $0.834 \AA$ Apix at $105,000 x$ magnification and $1.346 \AA$ pix at 64,000x magnification, which correspond to Nyquist limits of $1.668 \AA$ and $2.692 \AA$, respectively. Although our analyses here focus on apoferritin, we also collected 4 data sets from the much smaller aldolase to confirm the generality of our observations (Table 1).

\section{Influence of dose rates for imaging samples in CDS mode}

We first explored the influence of dose rates on imaging frozen biological samples recorded in CDS mode through a series of experiments, ranging from 4eps to 16eps. The resolutions of $3 \mathrm{D}$ reconstructions determined from these data sets were evaluated in terms of both absolute value in angstroms and as a percentage of the corresponding physical Nyquist frequency limit (Figure 1A, Table S3). Comparing apoferritin data sets collected at $105 \mathrm{kx}$, the one collected at a dose rate of 8eps reached a higher resolution than the ones at 4 and 16eps. The reconstruction from 200,000 particles yielded an average resolution of $1.75 \AA$, $\sim 95 \%$ of the physical Nyquist resolution, as compared to 1.78 $\AA$ from 4 and 16eps data sets. In addition, the benefit of collecting at 8 eps became even more pronounced when subsets of particles were used. A reconstruction from 10,000 particles reached $1.98 \AA$, $~ 84 \%$ Nyquist frequency at 8eps, but only $2.16 \AA$, $\sim 77 \%$ (8\% lower), at 16eps. Consistently, when comparing data subsets collected at a lower magnification, 64kx, 8eps data yielded higher resolutions than 16eps. However, these differences vanished when using 200,000 particles, for which both low magnification reconstructions reached $1.91 \AA$, an astonishing $\sim 141 \%$ of the physical Nyquist (Figure 1A, Table S3). 
bioRxiv preprint doi: https://doi.org/10.1101/2020.11.08.372763; this version posted November 8, 2020. The copyright holder for this preprint (which was not certified by peer review) is the author/funder, who has granted bioRxiv a license to display the preprint in perpetuity. It is made available under aCC-BY 4.0 International license.

Sun et al., 08 Nov 2020 - preprint copy - BioRxiv

\begin{tabular}{|c|c|c|c|c|c|c|c|}
\hline \multicolumn{8}{|c|}{ Apoferritin $64,000 x, 9$-hole image shift data collection, $1.346 \AA ̊$ /pixel (counted) } \\
\hline Dose rate & Mode & \# stacks & Spot size & Total dose & \# frames & Sec/frame & Collection time \\
\hline 8 eps & CDS & 1032 & 5 & $69 \mathrm{e} / \mathrm{A}^{2}$ & 120 & 0.13 & $\sim 9.5$ hours \\
\hline 8 eps & nonCDS & 1082 & 5 & $69 \mathrm{e} / \mathrm{A}^{2}$ & 120 & 0.13 & $\sim 10$ hours \\
\hline 16 eps & CDS & 912 & 4 & $69 \mathrm{e} / \mathrm{A}^{2}$ & 120 & 0.065 & $\sim 9$ hours \\
\hline $16 \mathrm{eps}$ & nonCDS & 860 & 4 & $69 \mathrm{e} / \mathrm{A}^{2}$ & 120 & 0.065 & $\sim 9$ hours \\
\hline \multicolumn{8}{|c|}{ Apoferritin 105,000x, 9-hole image shift data collection, $0.834 \AA ̊$ Ápixel (counted) } \\
\hline Dose rate & Mode & \# stacks & Spot size & Total dose & \# frames & Sec/frame & Collection time \\
\hline 4 eps & CDS & 1081 & 6 & $69 \mathrm{e} / \mathrm{A}^{2}$ & 120 & 0.1 & $\sim 9$ hours \\
\hline 8 eps & CDS & 1035 & 5 & $69 \mathrm{e} / \mathrm{A}^{2}$ & 120 & 0.05 & $\sim 8$ hours \\
\hline 8 eps & nonCDS & 1029 & 5 & $69 \mathrm{e} / \mathrm{A}^{2}$ & 120 & 0.05 & $\sim 8$ hours \\
\hline $16 \mathrm{eps}$ & CDS & 1142 & 4 & $69 \mathrm{e} / \mathrm{A}^{2}$ & 120 & 0.025 & $\sim 7$ hours \\
\hline
\end{tabular}

\begin{tabular}{|c|c|c|c|c|c|c|}
\hline \multicolumn{7}{|c|}{ Aldolase $64,000 \times$ x, 9-hole image shift data collection, 1.346 Å/pixel (counted) } \\
\hline Dose rate & Mode & \# of stacks & Spot size & Total dose & \# frames & Sec/ frame \\
\hline $8 \mathrm{eps}$ & CDS & 744 & 5 & $69 \mathrm{e} / \mathrm{A}^{2}$ & 120 & 0.13 \\
\hline $8 \mathrm{eps}$ & Non CDS & 696 & 5 & $69 \mathrm{e} / \mathrm{A}^{2}$ & 120 & 0.13 \\
\hline $16 \mathrm{eps}$ & CDS & 972 & 4 & $69 \mathrm{e} / \mathrm{A}^{2}$ & 120 & 0.065 \\
\hline $16 \mathrm{eps}$ & Non CDS & 810 & 4 & $69 \mathrm{e} / \mathrm{A}^{2}$ & 120 & 0.065 \\
\hline
\end{tabular}

Table 1. Data collection summary for apoferritin and aldolase data sets. All data sets were collected on a Titan Krios G3 at $300 \mathrm{kV}$ equipped with a Gatan K3 camera and BioQuantum energy filter. Applicable units are denoted in the tables and data sets are separated by pixel size.

While the gains in resolution when using a dose rate of 8eps were modest in terms of the global FSC resolution estimate, there were clear improvements in the maps. We scrutinized the structural features of each amino acid, but focused here on the atomic details of Trp-93 (Figure 1B-G). At 8eps, the four distal carbons of the benzene ring can be resolved to an extent that the ring has a clear hexagonal shape, unlike in the 16eps map where it appears more circular. Also, the hole in the tryptophan pyrrole ring becomes clearer as dose rate is decreased. Consistent with the numerical resolution, the hole in the pyrrole ring is not resolved in the lower magnification reconstructions. Maps reconstructed from only 30,000 particles show similar quality trends to those observed with all the particles. For example, the density around the backbone carbonyl directly behind Trp-93 is notably better defined in the high magnification, 8eps map than in the other 30,000 particle maps.

\section{Influence of CDS mode under conditions of constant coincidence loss}

Perhaps one of the most important new features on the $\mathrm{K} 3$ camera is the availability of CDS mode. To disentangle the conflicting influences of decreased noise and increased coincidence loss that are introduced when using CDS mode, we compared data collected such that the total coincidence losses, in principle, were held constant (Table S2). Specifically, apoferritin samples were exposed at 8eps CDS vs 16eps nonCDS at $64 \mathrm{kx}$ magnification, and 4 eps CDS vs 8 eps nonCDS at $105 \mathrm{kx}$ magnification.

Figure 2 summarizes our results for apoferritin and shows that operating in CDS mode produced higher quality data and yielded higher resolution reconstructions at both magnifications. At $64 \mathrm{kx}$ magnification, the average resolution of apoferritin from 200k particles is $1.91 \AA$ ( $141 \%$ of the Nyquist frequency) at 8eps in CDS mode, whereas at 16eps in nonCDS mode, the average resolution decreased by $7 \%$ to $1.98 \AA$. Smaller subsets of particles also reflect this trend, with the 8eps reconstructions improving $\sim 7-12 \%$ in terms of percentage of Nyquist frequency for each subset. At $105 \mathrm{kx}$ magnification, we observed $\sim 5 \%$ 
Sun et al., 08 Nov 2020 - preprint copy - BioRxiv

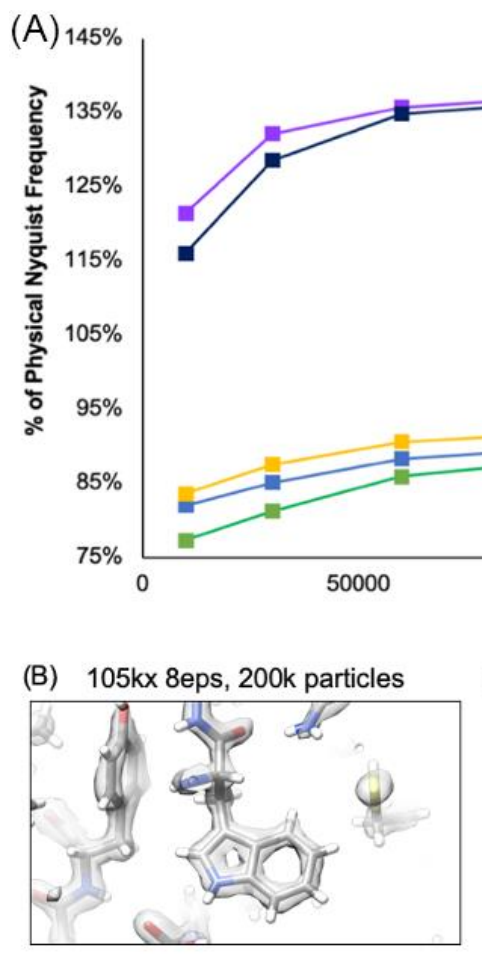

(E) $105 \mathrm{kx} 8 \mathrm{eps}, 30 \mathrm{k}$ particles

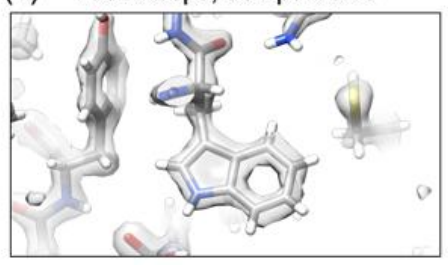

(F) $105 \mathrm{kx}$ 16eps, 30k particles

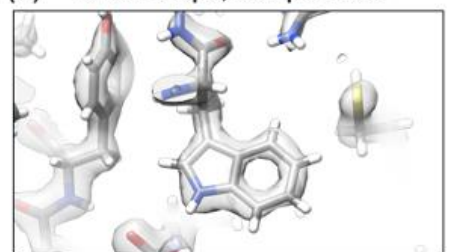

gain in resolution with 200k particles (Figure 2, Table S4). There is less separation between 4eps in CDS mode and 8eps in nonCDS mode at higher magnification, $3-5 \%$ for all subsets.

\section{Influence of CDS mode on imaging samples at constant dose rate}

Under conditions of constant coincidence loss, the gain in using CDS was clear, however, this led to practical differences in exposure time. Next, we compared data collected at a constant dose rate, 8eps, in CDS and nonCDS mode. Again, comparison of the 3D reconstructions showed that gains from CDS mode compensated for increased coincidence losses at both magnifications, and that the benefit was most prominent at higher magnification (Figure 3, Table S5). At $105 \mathrm{kx}$ magnification, the achievable resolution with CDS at 8eps was $10 \%$ higher than without CDS, $1.75 \AA$ compared to $1.94 \AA$ when comparing reconstructions from 200,000 particles each. This trend held for all particle subsets sampled at high magnification, with differences from 5$10 \%$ of percentage Nyquist. At $64 \mathrm{kx}$ magnification, the gain in resolution when using CDS mode was only $2 \%$. Compared with the gain from using CDS mode at constant coincidence loss (Figure 2), the gain at $105 \mathrm{kx}$ magnification from using CDS mode at constant dose rate was similar (Figure 3), while it was reduced for the 64kx magnification data sets.
Figure 1. Influence of dose rate on reconstructions of apoferritin from data recorded on the $\mathrm{K} 3$ detector. (A) Results of 3D reconstructions from apoferritin data sets collected at different dose rates. The results are plotted as a function of percent of physical Nyquist frequency versus particle numbers in order to compare the effect of dose rate on data sets collected at different magnifications. $105 \mathrm{kx}$ correlates to a physical pixel size of $0.834 \AA$ A $/$ ix and $64 \mathrm{kx}$ to $1.346 \AA$ A $/$ ix . The highest resolution achieved for each data set is labelled. (B-D) CryoEM maps calculated from 200k particles for (B) 8eps (C) 16eps data sets collected at $105 \mathrm{kx}$ magnification and (D) 8eps collected at $64 \mathrm{kx}$ magnification. (E-G) 3D reconstructions calculated from 30,000 particles for (E) 8eps and (F) 16 eps data sets collected at $105 \mathrm{kx}$ magnification, and (G) 8eps collected at $64 \mathrm{kx}$ magnification. Data was processed in RELION v3.1, following standard protocols and sharpened using standard RELION post-processing protocols. These snapshots allow us to visualize the higher resolution structural information retained in the data collected at lower dose rates.

\section{Imaging aldolase with and without CDS mode}

As a check on the generality of our apoferritin observations, we also explored the influence of dose rate and CDS mode on aldolase, which is smaller $(\sim 150 \mathrm{kDa})$ and has less symmetry (D2). Unlike the apoferritin sample, aldolase was used as purchased rather than expressed and purified in-house, a fact that potentially limits the ultimate achievable resolution. Consistent with the apoferritin data sets, collecting at 8eps outperformed 16eps in terms of achievable Nyquist frequency. Lower dose rate yielded higher resolution reconstructions across particle subsets, with a larger difference being seen when fewer particles were used. Reconstructions from 10,000-particle subsets reached $3.5 \AA$ (77\%) at 8 eps vs. $3.8 \AA(71 \%$ ) at 16eps (Figure S6 purple and orange, Table S8).

Surprisingly, the gain from using CDS mode when coincidence loss was constant was trivial compared with apoferritin. Reconstructions from data collected at 8eps with CDS yielded nearly the same resolutions as 16eps without CDS across the different subsets (Figure S6 purple and yellow curves, Table S8). By contrast, at fixed dose rates, CDS was always comparable or better than without CDS (Figure S6 purple vs pink or red vs orange, Table S8). For data collected at 8eps, CDS mode is 5$10 \%$ better than nonCDS with the 200,000 particle subsets reconstructing to $2.7 \AA$ (CDS) and $2.8 \AA$ (nonCDS), respectively. Thus, the overall trends seen for apoferritin held for aldolase although some confounding 
bioRxiv preprint doi: https://doi.org/10.1101/2020.11.08.372763; this version posted November 8, 2020. The copyright holder for this preprint (which was not certified by peer review) is the author/funder, who has granted bioRxiv a license to display the preprint in perpetuity. It is made available under aCC-BY 4.0 International license.

Sun et al., 08 Nov 2020 - preprint copy - BioRxiv

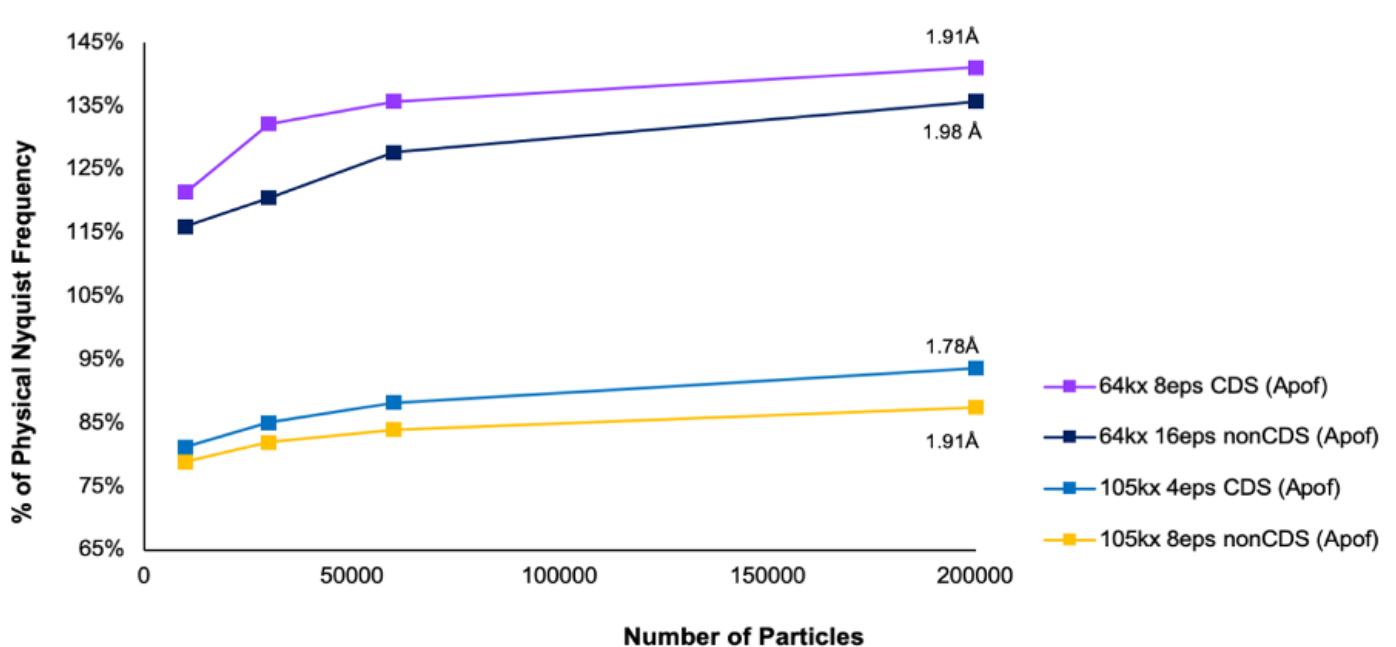

Figure 2. Benefits of imaging apoferritin in CDS mode when comparing data sets with equivalent coincidence loss of electron events. Comparing results of apoferritin in CDS and nonCDS modes where coincidence loss events are consistent. These results are plotted as a function of percent of physical Nyquist frequency versus particle number and highest achievable resolutions are labelled. Data was processed in RELION v3.1, following standard protocols (Scheres, 2012; Zivanov et al., 2018)

For apoferritin, five minutes of data collection yielded the necessary number of particles to reconstruct below $3 \AA$ (Figure 4). Interestingly, comparing the two magnifications a crossover point was observed where it became more advantageous to image apoferritin at high magnification. This crossover point was at $\sim 2.2 \AA$ and between $20-60$ minutes of data collection time. The data indicate that imaging at a $64 \mathrm{kx}$ magnification reaches $2.5 \AA$ resolution $\sim 5 \mathrm{x}$ faster than imaging at $105 \mathrm{kx}$ magnification. Thus, an intermediate resolution data collection session could last as little as 30 minutes at $64 \mathrm{kx}$ magnification, not including automated data collection set-up, for this benchmark sample. To reach high resolutions, below $2 \AA$ for apoferritin, a higher magnification should be used and the data collection session will need to be at least 2.5 hours (Figure 4). If octahedral symmetry is not applied, making the sample more comparable to other large but asymmetric particles, a data collection of around 8 hours would be necessary to reach sub-2 $\AA$ resolution (Figure S3).

\section{Maximal resolutions attainable with these data and atomic modelling}

To eliminate the effects of unexplored higher-order optical aberrations and astigmatisms that were present in the dataset, we performed three rounds of CTF refinements for all the apoferritin and aldolase data sets: refining magnification anisotropy, then refining third and fourth-order optical aberrations, and finally refining per-particle

Figure 3. Practical consideration of imaging apoferritin using CDS mode at our recommended dose rate of 8eps. Comparing results of aldolase in CDS and nonCDS modes where coincidence loss events are consistent. Resolution estimations were made following the FSC 0.143 criteria (Rosenthal \& Henderson, 2003) and results are plotted as a function of percent of physical Nyquist frequency versus particle number. The highest achievable resolution is labelled. Data was processed in RELION v3.1, following standard protocols (Scheres, 2012; Zivanov et al., 2018). 
bioRxiv preprint doi: https://doi.org/10.1101/2020.11.08.372763; this version posted November 8, 2020. The copyright holder for this preprint (which was not certified by peer review) is the author/funder, who has granted bioRxiv a license to display the preprint in perpetuity. It is made available under aCC-BY 4.0 International license.

Sun et al., 08 Nov 2020 - preprint copy - BioRxiv

defocus and per-micrograph astigmatism. With these, we far surpassed the physical Nyquist limit of $2.69 \AA$ for apoferritin data collected at $64 \mathrm{kx}$ magnification $(1.9 \AA$ ) and reached $1.75 \AA$, $95 \%$ of the physical Nyquist

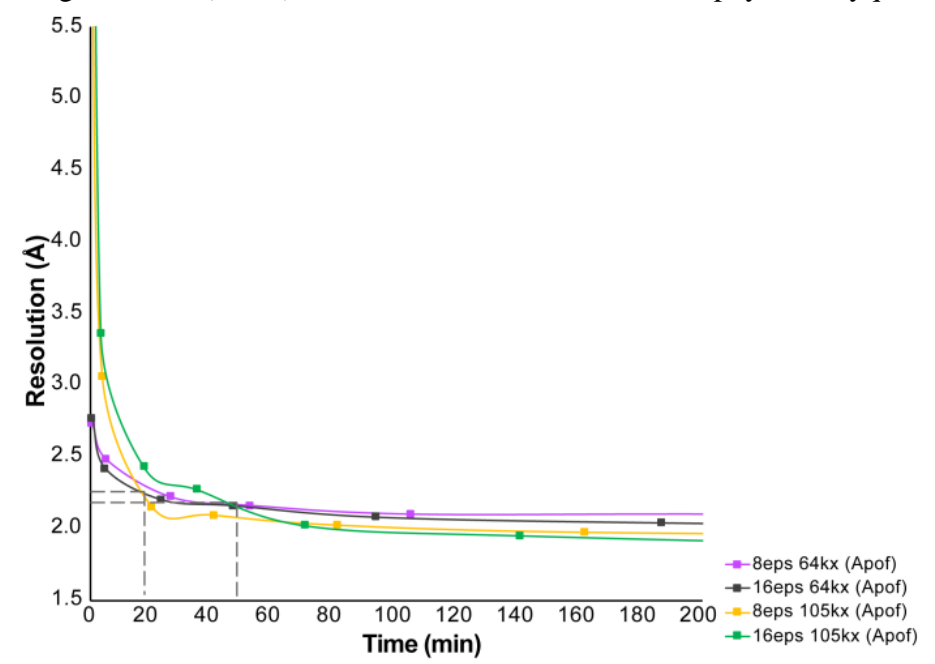

Figure 4. Time versus resolution plot for apoferritin refined with octahedral symmetry. $35 \%$ of particles were selected from each image to determine the time it takes different data collection conditions to reach a certain resolution. There is a crossover point where a smaller pixel size should be utilized to reach the highest resolutions, $<2.2 \AA$ resolution, between 15- and 50-min depending on dose rate. Data was processed in cryoSPARC v2.12.4 4 (Punjani et al., 2017), following standard protocols without 3D classification.

limit of $1.69 \AA$ at $105 \mathrm{kx}$ magnification, using 200,000 particles.

To further optimize motion correction for the data sets collected at the optimal dose rate, 8eps, we performed Bayesian polishing, followed by 3D auto refinement (Figure S4 and Table S7). These steps improved the overall resolution of the reconstructions collected at all magnifications and in both CDS and nonCDS mode, as expected. It is worth noting that for the highest resolution reconstructions from $200 \mathrm{k}$ and $571 \mathrm{k}$ particles at $105 \mathrm{kx}$ magnification, polishing improved the resolution to $1.69 \AA$ and $1.65 \AA$, respectively. Similarly, polishing improved the resolution at $64 \mathrm{kx}$ magnification from $1.91 \AA$ to $1.87 \AA$ (200k particles) and from $1.86 \AA$ to $1.80 \AA$ ( $373 \mathrm{k}$ particles). The latter corresponds to $149 \%$ of the physical Nyquist resolution, indicating the remarkable benefits of super-resolution in CDS mode.

For fitting the atomic model at this resolution, we adopted high-resolution refinement strategies from traditional x-ray crystallography and performed such refinements with individual b-factor refinements in reciprocal space. In such, we were able to fit each atom, including hydrogens, with appropriate per-atom B-factors. When viewing our highest resolution map, density for hydrogen atoms is clearly visible on both the alpha carbon backbone and some side chains (Figure $5 \mathrm{D}, \mathrm{E}, \mathrm{I}, \mathrm{J})$. They are better resolved at $1.65 \AA$ as expected. In fact, some hydrogens are more visible in our cryo-EM maps than maps simulated at $1.5 \AA$ and $1.3 \AA$ resolution would predict (Figure 5A-C, F-H).

\section{Discussion}

In this study, we experimentally evaluated Gatan K3 camera performance as a function of dose rate, magnification and CDS mode using the standard sample, apoferritin. In CDS mode, imaging at 8 electron per pixel per second (eps) was always better or as good as imaging at 16eps (Figure 1A and Figure S3), which was expected based on coincidence loss effects. While, surprisingly, reducing the dose rate to 4 eps provided no additional benefit and instead slightly worsened obtainable resolutions. With increasing dose rate, the DQE will decrease, especially at low frequencies. This is largely due to a depressed NPS and MTF whereas higher resolutions are largely unaffected. Comparing reconstructions collected at 8eps and 16eps, the differences in resolutions were greater at the $105 \mathrm{kx}$ magnification, than at the $64 \mathrm{kx}$ magnification. This suggests that increased coincidence loss affects final reconstruction resolutions most by down weighting intermediate frequencies that are
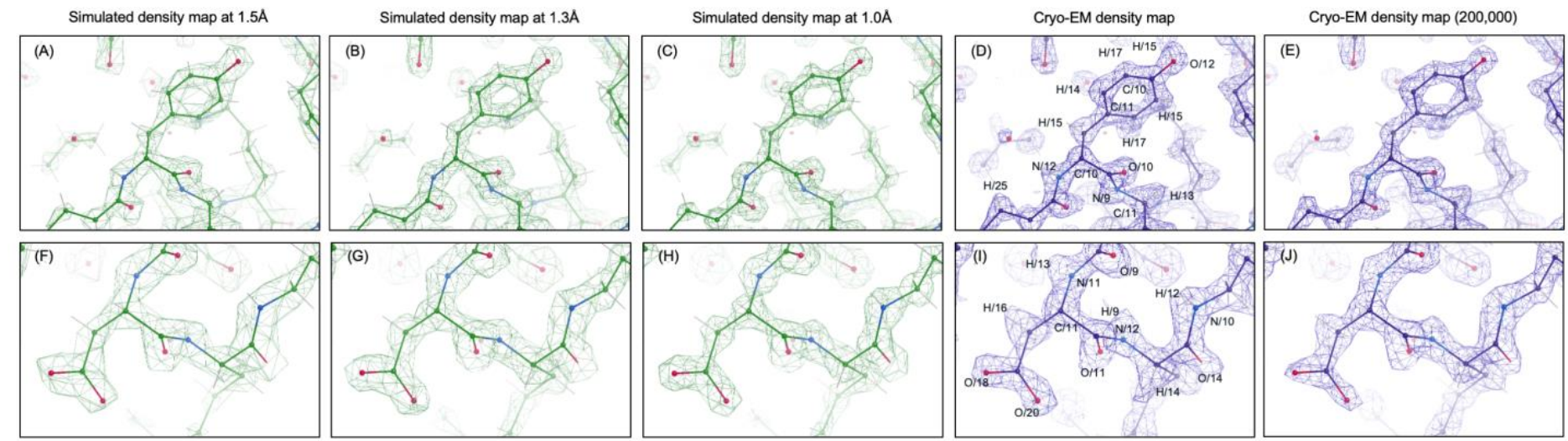

Figure 5. Structural features of apoferritin cryo-EM density map at a global resolution of 1.65 A. Two apoferritin residues, Trp-168 (A-E) and Asp171 (F-J) are selected for comparison among our cryo-EM reconstructions from different number of particles (reconstruction from 500k particles (D) and (I); reconstruction from $200 \mathrm{k}$ particles $(\mathrm{E})$ and $(\mathbf{J})$ ) and simulated density maps at the indicated resolution (A-C and F-H). The refined atomic coordinates are fitted into each density map and shown as stick and ball. Density that can be attributed to hydrogen atoms, specifically HB2/ Trp 168 (assigned bfactor $\sim 15$ ) and HD1 Trp 168 (assigned b-factor 14) in tops panels and HB2/Asp 171 (assigned b-factor $\sim 16$ ) and H/ Asp 171 (assigned b-factor $\sim 13$ ) in bottom panels, can be clearly observed in our cryo-EM density map, in $1.0 \AA$ (C and $\mathbf{G}$ ) and $1.3 \AA$ (B and F) simulated maps, but are barely seen in the $1.5 \AA$ simulated map (A and E). Maps simulations were carried out in Phenix (Adams et al., 2010) using phenix.fmodel and phenix.mtz2mrc functions, which consider per-atom $b$ factors and generate maps at different resolutions. Estimated per atom b-factors were labelled in 5D and 5I. Cryo-EM data was collected at $105 \mathrm{kx}$, 8eps, CDS mode using 9-hole image shift data collection scheme. Figures were prepared in Coot ((Emsley \& Cowtan, 2004)) software. 
Sun et al., 08 Nov 2020 - preprint copy - BioRxiv

well within physical Nyquist (Li, Zheng et al., 2013). By contrast with coincidence loss, the expectation is that CDS mode would enhance DQE across all resolution ranges. Influences of CDS mode at different coincidence loss rates were characterized by comparing reconstructions from apoferritin data sets collected at the same coincidence loss rate or same read-out dose rate ( $2 \mathrm{x}$ difference in terms of coincidence loss rate). Our results validated that operating in CDS mode would further reduce noise and improve DQE and in such, compensates for any increased coincidence losses, in general (Figure 2 and Figure 3).

There have been many discussions as how close obtainable resolutions can be to those imposed by the physical Nyquist sampling. Of concern are both aliasing artifacts, where higher resolution information becomes folded into lower resolution observations, as well as optimal utilization of the camera DQE that falls off as physical Nyquist is approached. The advent of super-resolution sub-pixel localization on the K2 camera provided a straightforward approach to avoiding aliasing. However, the DQE at physical Nyquist remains low, significantly diminishing information transfer beyond this point. Indeed, most efforts to obtain high resolution reconstructions of apoferritin have used physical pixel sizes of $0.46 \AA$ or smaller (Nakane et al., 2020; Yip et al., 2020; Zhang et al., 2020).

Fortunately, the many improvements in the $\mathrm{K} 3$ camera significantly increase DQE performance at Nyquist frequency, forcing us to reconsider the true utility of super-resolution information. This is clearly evident in the apoferritin data collected at $64 \mathrm{kx}$ magnification. Using 8eps and CDS mode, we readily obtained $1.91 \AA$ or $141 \%$ of the physical Nyquist limit $(2.69 \AA)$ with $200 \mathrm{k}$ particles. Using a smaller subset, of 30k particles, reached $132 \%$ of Nyquist, whereas the full set of good particles (373k) combined with Bayesian polishing resulted in a final resolution of $1.80 \AA$, corresponding to a stunning $149 \%$ of the physical Nyquist. This indicates that the increased DQE now available in the K3, especially when using CDS mode, can significantly benefit data collected at lower magnification than typically utilized for highresolution cryo-EM.

A parallel study on aldolase validated the benefit of using CDS mode for high-resolution cryo-EM reconstruction determination and are largely consistent with apoferritin with a few notable differences. As expected, collecting data at a higher dose rate consistently worsened achievable resolution, but the negative effects of increasing dose rates were larger than observed with apoferritin. (Figure S6). When dose rates were matched, CDS clearly outperformed nonCDS mode for both apoferritin (Figure 3) and aldolase (Figure S6). However, when the coincidence loss rate remained constant, CDS only had a trivial impact on the aldolase and seemed unable to overcome the loss from increased coincidence events as it does for apoferritin. This is likely because coincidence losses result in an amplitude decrease at resolutions lower than half of the physical Nyquist limit, and such resolution may dominate the particle alignment accuracy for smaller particles like aldolase.

As to the goal of balancing collection time and resolution, our data showed that imaging at a larger pixel size allowed us to reach intermediate resolutions $\sim 5 \mathrm{x}$ faster than imaging at a higher magnification (Figure 4). This holds true in our imaging scheme because we acquire one image per hole. The benefit of more particles per image would be offset when taking multiple images per hole (Weis \& Hagen, 2020). Because reaching intermediate resolutions appears faster at lower magnification, routine collection at a lower magnification would be most desirable when large numbers of particles are required, such as when determining multiple structures of heterogenous samples. By contrast, if the hypothesis being tested can only be addressed with a very highresolution structure, such as locating the binding site and position of a small molecule or resolving disease mutations traced to single amino acids, high magnification and physical pixel sizes below $1 \AA$ would be preferred.

In summary, high (physical pixel below $1 \AA$ ) or low magnification (physical pixel above $1 \AA$ ) should be selected based on the biological problem being asked, noting that reconstructions surpassing the physical Nyquist are now readily achievable when using a larger pixel size. At either magnification, we would advise users to routinely use an intermediate dose rate of 8eps in CDS mode and a 9-hole image shift data collection method to achieve both high-resolution reconstructions and high-throughput collections.

\section{Materials and Methods}

\section{Plasmid and constructs of heavy chain mouse apoferritin}

Plasmid: pET24a-mFth1 (full-length mouse Ferritin heavy chain w/o tag): AAGTGGCGAGCCCGATCTTCCCCATCGGTGATGTCGGCGATATAGG CGCCAGCAACCGCACCTGTGGCGCCGGTGATGCCGGCCACGATGC GTCCGGCGTAGAGGATCGAGATCTCGATCCCGCGAAATTAATACGA CTCACTATAGGGGAATTGTGAGCGGATAACAATTCCCCTCTAGAAAT AATTTTGTTTAACTTTAAGAAGGAGATATACATATGACCACCGCGTCT CCCTCGCAAGTGCGCCAGAACTACCACCAGGACGCGGAGGCTGCC ATCAACCGCCAGATCAACCTGGAGTTGTATGCCTCCTACGTCTATCT GTCTATGTCTTGTTATTTTGACCGAGATGATGTGGCTCTGAAGAACT TTGCCAAATACTTTCTCCACCAATCTCATGAGGAGAGGGAGCATGCC GAGAAACTGATGAAGCTGCAGAACCAGCGAGGTGGCCGAATCTTCC TGCAGGATATAAAGAAACCAGACCGTGATGACTGGGAGAGCGGGCT GAATGCAATGGAGTGTGCACTGCACTTGGAAAAGAGTGTGAATCAG TCACTACTGGAACTGCACAAACTGGCTACTGACAAGAATGATCCCCA CTTATGTGACTTCATTGAGACGTATTATCTGAGTGAACAGGTGAAAT CCATTAAAGAACTGGGTGACCACGTGACCAACTTACGCAAGATGGG TGCCCCTGAAGCTGGCATGGCAGAATATCTCTTTGACAAGCACACC CTGGGACACGGTGATGAGAGCTAACTCGAGCACCACCACCACCACC ACTGAGATCCGGCTGCTAACAAAGCCCGAAAGGAAGCTGAGTTGGC TGCTGCCACCGCTGAGCAATAACTAGCATAACCCCTTGGGGCCTCT AAACGGGTCTTGAGGGGTTTTTTGCTGAAAGGAGGAACTATATCCG GAT

\section{Protein Expression and Purification}

\section{Heavy chain mouse apoferritin}

Murine heavy chain ferritin in pET24a plasmid was gifted (M. Kikkawa), amplified in DH5a, and sequenced (ElimBio). One liter of E. coli BL21(DE3) was grown in LB with $50 \mathrm{ug} / \mathrm{mL}$ kanamycin and $0.5 \%$ glucose to $\mathrm{OD}_{600}$ $0.75 .1 \mathrm{mM}$ IPTG was added for $2.5 \mathrm{hr}$ at $37^{\circ} \mathrm{C}$. Cells were harvested, washed in phosphate buffered saline, and resuspended in $30 \mathrm{mM} \mathrm{HEPES}$ $\mathrm{pH} 7.5,300 \mathrm{mM} \mathrm{NaCl}, 1 \mathrm{mM} \mathrm{MgSO} 4,1 \mathrm{mg} / \mathrm{mL}$ lysozyme, and an EDTAfree protease inhibitor tab (Sigma). The resuspension was sonicated for 150 seconds at $50 \%$ power (120-watt Active Motif). Cell debris was 
Sun et al., 08 Nov 2020 - preprint copy - BioRxiv

centrifuged at $20,000 \times g$ for 30 minutes at $4^{\circ} \mathrm{C}$. The supernatant was incubated at $70^{\circ} \mathrm{C}$ with gentle nutation. The sample was centrifuged at $20,000 \times g$ for 30 minutes at $4^{\circ} \mathrm{C}$. $6.65 \mathrm{~g}$ of $\left(\mathrm{NH}_{4}\right)_{2} \mathrm{SO}_{4}$ was added to $20 \mathrm{~mL}$ of the supernatant on ice and stirred for 10 minutes. The sample was centrifuged at $14,000 \mathrm{xg}$ for 20 minutes and the pellet was resuspended in $2 \mathrm{~mL} 20 \mathrm{mM}$ HEPES pH 7.5, $300 \mathrm{mM} \mathrm{NaCl}$. The sample was then dialyzed against $20 \mathrm{mM} \mathrm{HEPES} \mathrm{pH} \mathrm{7.5,300} \mathrm{mM} \mathrm{NaCl}$ overnight at $4^{\circ} \mathrm{C}$ in a 20,000 MWCO Slide-A-Lyzer (ThermoFisher). The sample was recovered from the dialysis cassette and centrifuged at $55,000 \mathrm{xg}$ for 15 minutes at $4^{\circ} \mathrm{C}$. The supernatant was concentrated to $\sim 1 \mathrm{~mL}$ in a $50 \mathrm{MWCO}$ spin column (Millipore). The final concentrated sample was centrifuged at 55,000 $\mathrm{xg}$ for 15 minutes at $4^{\circ} \mathrm{C}$. An $\mathrm{A}_{280}$ reading was taken in order to not overload the Superose $6 / 150$ Increase column. The sample was run over a the Superose $6 / 150$ Increase column in two aliquots at $0.4 \mathrm{ml} / \mathrm{min}$ in $20 \mathrm{mM}$ HEPES pH 7.5, $300 \mathrm{mM} \mathrm{NaCl}$. Peak fractions were saved and frozen at $80^{\circ} \mathrm{C}$ in aliquots at $13 \mathrm{mg} / \mathrm{mL}$ (first run) and $6 \mathrm{mg} / \mathrm{mL}$ (second run) as determined by nanodrop $A_{280}$ readings.

\section{Rabbit aldolase}

Rabbit aldolase was purchased from Sigma-Aldrich (A2714-100UN, lot\# SLBR7752V) in powdered form. Sample was dissolved at $1.6 \mathrm{mg} / \mathrm{mL}$ in 20 mM HEPES ( $\mathrm{pH} 7.5$ ), $50 \mathrm{mM} \mathrm{NaCl}$ and used directly.

\section{Cryo-EM Grid Preparation}

To closely mimic standard sample preparation practices in the facility, all apoferritin and aldolase samples were frozen on 300-mesh Quantifoil gold holey carbon grids with hole size and spacing of 1.2/1.3 $\mu \mathrm{m}$ (Quantifoil) using a Mark IV vitrobot (ThermoFisher). Freezing details described as the followings.

\section{Apoferritin}

$6 \mathrm{mg} / \mathrm{mL}$ apoferritin aliquots were thawed from storage at $-80^{\circ} \mathrm{C}$ and centrifuged at $55,000 \mathrm{xg}$ for 15 minutes at $4^{\circ} \mathrm{C} .2 \mu$ apoferritin was applied to glow-discharged gold holey carbon 1.2/1.3 300-mesh grids (Quantifoil). Grids were blotted for 4 seconds at -2 force and vitrified in liquid ethane using a MarkIV Vitrobot (ThermoFisher). The blotting chamber was maintained at $22^{\circ} \mathrm{C}$ and $100 \%$ humidity during freezing.

\section{Aldolase}

$0.8 \mathrm{mg} / \mathrm{mL}$ aldolase solution was applied to glow-discharged gold holey carbon 1.2/1.3-200 mesh grids (Quantifoil). Grids were blotted for 3 seconds at 0 force and vitrified in liquid ethane using a MarkIV Vitrobot (ThermoFisher). The blotting chamber was maintained at $6^{\circ} \mathrm{C}$ and $100 \%$ humidity during freezing.

\section{Cryo-EM Data Collection}

Briefly, all images were acquired on a Titan Krios microscope outfitted with a Gatan K3 detector and BioQuantum Imaging Filter operating in nanoprobe and EF-TEM mode, a C2 aperture size of $70 \mu \mathrm{m}$, an objective aperture size of $100 \mu \mathrm{m}$, and an energy filter slit width of $20 \mathrm{eV}$. Data was collected automatically using $3 \times 3$ 9-hole image shift application in SerialEM, with the same defocus range, total electron dose, and frame number with a constant electron dose per frame. Details are described as the followings.

\section{Microscope alignment}

Full alignments of the microscope were performed only after K3 installation, after which only direct alignments were routinely checked before each data collection. On a Titan Krios microscope, using a $70 \mu \mathrm{m}$ C2 aperture, the sample was first set to a stage position at eucentric height over carbon on a grid square that appeared free of defects at lower magnification. Specimen was then brought close to focus and astigmatism was adjusted before proceeding with beam alignment. First, pivot points and rotation center were roughly adjusted at imaging magnification (or higher at nominal $165,000 x$ for rotation center). Further tuning of pivot points and rotation center were then performed by iterating through microscope coma-free pivot point and coma-free alignment procedures for four directions of beam tilt. Finally, SerialEM implementation of astigmatism and Coma-free alignment correction by CTF fitting was used for fine adjustment, correcting 8 beam tilt directions. Beam intensity was adjusted to a diameter within parallel illumination conditions for a 3condenser lens system, and gave the appropriate dose for the dataset (spot size varying between 4 and 5). SerialEM Coma vs. Image Shift procedure was then performed for each of these dose settings accounting for any differences in illumination and saved as separate settings files.

\section{BioQuantum Gatan energy filter tuning}

The BioQuantum Gatan energy filter was tuned once a week, without sample in the beam path. We found that within a week for our GIF values for isochromaticity, achromaticity, magnification and distortion had almost no change (within $1 \mathrm{eV}$ for isochromaticity). tuning was performed by decreasing spot size to 1 which would give sufficient dose for full GIF tuning to yield good results and uniform slit edge. For zero-loss peak centering, a dose around 16 eps was sufficient and consistent within $5 \mathrm{eV}$ over the course of a week. A slit width of $20 \mathrm{eV}$ was used for data collection. A 100 $\mu \mathrm{m}$ objective aperture was then inserted and centered before a final astigmatism adjustment, after which the microscope was ready for data collection.

\section{Considerations of data collection consistency}

For consistency, apoferritin datasets were collected in one imaging session, over the course of four days, on a single grid. Aldolase datasets were collected in two sessions from grids vitrified under the same conditions in the same freezing session. A gain reference was collected in CDS and nonCDS mode for each magnification and dose rate.

\section{Image processing}

During data collection, images were pre-processed to provide feedback on image quality. All pre-processing was carried out using Scipion software (de la Rosa-Trevín et al., 2016). Beam-induced motion correction and dose weighing were performed on the raw movie stacks using MotionCor2 (Zheng et al., 2017) using a $7 \times 5$ patch size and a b-factor of 100 with 7 iterations. Super-resolution movies were saved for further analysis. Wholeimage contrast transfer function (CTF) estimation was performed using CTFFFIND 4 (Rohou \& Grigorieff, 2015).

\section{Apoferritin data collected at 105,000x magnification}

For the data sets of apoferritin, collected at $105,000 \times$ magnification, dose rates of 4 and 8 and 16 eps, image processing was performed in RELION 3.1 (Zivanov et al., 2018; Scheres, 2012) software following the standard protocols. Particles were picked using the Laplacian-of-gaussian algorithm and extracted at $4 x$ binned. These particles were subjected to standard 3D classification, to eliminate any unfit classes. The selected good class was re-extracted at $1 \mathrm{x}$ binned with a box size of 512 pixels and subjected to standard 3D auto-refinement to give the initial reconstruction. Subsequently, three runs of CTF refinement were performed using default parameters: first refining magnification anisotropy; then refining optical aberrations (up to the 4th order); and finally refining per-particle defocus and per-micrograph astigmatism. Another round of 3D auto-refinement with the refined values were performed.

High-resolution structure of apoferritin 
bioRxiv preprint doi: https://doi.org/10.1101/2020.11.08.372763; this version posted November 8, 2020. The copyright holder for this preprint (which was not certified by peer review) is the author/funder, who has granted bioRxiv a license to display the preprint in perpetuity. It is made available under aCC-BY 4.0 International license.

Sun et al., 08 Nov 2020 - preprint copy - BioRxiv

To further improve the resolution of the optimal data set, the one collected with a dose rate of 8eps at 105,000x magnification at CDS mode, the standard Bayesian polishing (Zivanov et al., 2019) was performed, followed by standard 3D auto-refinement. Then another round of CTF refinement was performed as it's described earlier. These yield a final average resolution of $1.66 \AA$ ( $F S C=0.143$ ), with an estimated accuracy aligned angles 0.325 degrees and offsets $=0.182969$ Angstroms.

\section{Apoferritin data collected at $64,000 x$ magnification}

For the data sets of apoferritin collected at $64,000 x$ magnification, dose rates of 8 eps under CDS and standard nonCDS modes, particles were picked using Gautomatch (Kai Zhang, https://www.mrcImb.cam.ac.uk/kzhang/). The following image processing was performed in RELION 3.1 (Zivanov et al., 2018; Scheres, 2012) software using the same protocols described above.

For the data sets of apoferritin collected at 64,000 magnification, dose rates of 16 eps under CDS and standard non-CDS modes, image processing was performed using cryoSPARC 2.14.2 (Punjani et al., 2017) following standard protocols. Particles were picked using the template picking algorithm using preliminary $2 \mathrm{D}$ averages from a blob pick job performed on 10 micrographs. Putative particles were extracted and $8 x$ binned. 2D classification was performed to screen out ice contamination and clearly damaged particles. The remaining particles were subjected to $\mathrm{Ab}$ Initio Reconstruction with octahedral symmetry, to eliminate any unfit classes. The good class was re-extracted without binning at a box size of 300 pixels and subjected to standard Homogenous Refinement including Defocus Refinement and Global CTF Refinement using octahedral symmetry. All available higher-order magnification parameters were fit and corrected.

Final particle stack was exported using csparc2star.py (Asarnow, D. et al., 2019), masked, and postprocessed in RELION 3.1 (Zivanov et al., 2018; Scheres, 2012).

All reported resolutions were based on the 0.143 Fourier shell criterion (Chen et al., 2013; Rosenthal \& Henderson, 2003) with all Fourier shell correlation (FSC) curves corrected for the effects of soft solvent masking by high-resolution noise substitution (Chen et al., 2013) as calculated in RELION 3.1. Masks were generated in RELION at the $50 \%$ of the density maps' maximum density values with 8-pixel extension and 8-pixel width.

\section{Aldolase data collected at $64,000 \times$ magnification}

For the data sets of aldolases, collected at $\mathbf{6 4 , 0 0 0} \times$ magnification, dose rates of 8eps under CDS and standard nonCDS modes, image processing was performed in RELION 3.1 software following the same standard as for apoferritin data collected at $64,000 x$ magnification.

For the data sets of aldolases collected at $64,000 x$ magnification, dose rates of 16eps under CDS and standard non-CDS modes, image processing was performed using cryoSPARC 2.14.2 (Punjani et al., 2017) following the same standard as for apoferritin collected at $64,000 x$ magnification.

All reported resolutions are based on the 0.143 Fourier shell criterion (Chen et al., 2013; Rosenthal \& Henderson, 2003) with all Fourier shell correlation (FSC) curves corrected for the effects of soft solvent masking by high-resolution noise substitution (Chen et al., 2013). Masks were auto generated in cryoSPARC and postprocessing was run in RELION for consistency with data sets processed in RELION.

\section{Time to resolution plots}

Processing was carried out as above except particles were not subjected to a 3D classification step using Ab Initio Reconstruction job and perparticle defocus and local CTF refinement were not performed. These particles were selected, cleaned from obvious contamination and refined using standard Homogenous Refinement in cryoSPARC (Punjani et al., 2017) to achieve a reasonable resolution in the shortest period of time. For apoferritin refinements with octahedral symmetry, $\sim 35 \%$ of particles were selected using the Inspect Picks job in cryoSPARC because when using all particles in an image the resolution dropped too quickly to see any difference between different conditions. For apoferritin refinements with $\mathrm{C} 1$ symmetry and all aldolase refinements, all picked particles were used. Final resolutions are reported from the tight mask FSC curves reported from cryoSPARC 2.14.2 (Punjani et al., 2017).

\section{Atomic modelling}

The x-ray crystal structure PDB 5OBA was docked into the cryo-EM density map in UCSF Chimera (Pettersen et al., 2004) and the 24 chains were refined against full the cryo-EM density maps in Phenix software (Adams et al., 2010) following the recommended protocol for refining medium to high resolution structures. In reciprocal space refinement, the main refinement loops are as followings, (1) converting cryo-EM density map to structure factors using phenix.map_to_structure_factors function; (2) refinement with bulk-solvent and individual anisotropic refinement using phenix.refine function; (3) refinements with individual anisotropic refinement but without bulk-solvent refinement using phenix.refine function. In addition, during the individual anisotropic refinement, the hydrogens were refined as a riding model. The model was then further refined in real space with phenix.real_space_refine and subsequently adjusted manually in Coot (Emsley \& Cowtan, 2004). Validation was carried out using full validation function in Phenix. The full cryo-EM density map with intermediate $b$-factor sharpened applied, $b$ factor $=-25$, was used for phenix.map_to_structure_factors conversion and cryo-EM validation.

\section{Calibration and refinement of pixel sizes}

Gatan K3 pixel size was initially calibrated through an autocorrelation routine in SerialEM (Mastronarde, 2005) using a cross-line replica grating grid, finding correlation peaks progressively further out to estimate the spacing between peaks along two axes. Using this method, we estimated the pixel size value at both nominal magnifications of $105,000 x$ and 64,000 $x$ to be $0.853 \AA /$ pixel and $1.378 \AA /$ pixel initially. We then used the highresolution reconstructions of apoferritin to further refine the pixel size (Wu et al., 2020). The x-ray crystal structures of apoferritin were first docked and then refined into respective cryo-EM reconstructions of voxel sizes ranging from $0.416 \AA /$ pixel (counted $0.832 \AA /$ pixel) to $0.4265 \AA /$ pixel (counted $0.853 \AA / p i x e l$ ) for data collected at $105 \mathrm{kx}$ magnification, and from $0.671 \AA ̊ 2$ pixel (counted $1.342 \AA /$ pixel) to $0.689 \AA /$ pixel (counted $1.378 \AA$ A pixel) using phenix.real_space_refinement package (Adams et al., 2010). Meanwhile, the $x$-ray structure was also rigid-body fitted into the cryo-EM density maps in UCSF Chimera (Pettersen et al., 2004) By examining the map vs map $C C$ values reported from phenix and the $x$-ray simulated map vs cryo-EM CC values from Chimera, we found that at the pixel size values of $0.834 \AA /$ pixel and $1.346 \AA$ /pixel gave the highest score.

\section{Author Contributions and Notes}

M.S, C.M.A, E.T, D.A.A, K.A.V, Y.C, D.S, and A.F designed research, M.S, C.M.A, and E.T performed research, M.S, C.M.A, and E.T analyzed data; and M.S, C.M.A, E.T, D.A.A, K.A.V, Y.C, D.S, and A.F wrote and edited the paper.

The authors declare no conflict of interest. 
bioRxiv preprint doi: https://doi.org/10.1101/2020.11.08.372763; this version posted November 8, 2020. The copyright holder for this preprint (which was not certified by peer review) is the author/funder, who has granted bioRxiv a license to display the preprint in perpetuity. It is made available under aCC-BY 4.0 International license.

Sun et al., 08 Nov 2020 - preprint copy - BioRxiv

\section{Data availability}

The electron density maps and atomic models have been deposited into the Electron Microscopy Data Bank (EMDB) and the Protein Data Bank (PDB). The accession codes are EMD-22972 and 7KOD, respectively.

\section{Acknowledgments}

We thank the Kikkawa lab for the murine heavy-chain ferritin construct plasmid; David Bulkley and Glenn Gilbert for support in data collection and microscope management through the University of California San Francisco Advanced Cryo-Electron Microscopy Facility; Matt Harrington, Joshua Baker-LePain, and the QB3 shared cluster (NIH grant 1S10OD021596-01) for high-performance computing (HPC) support; Paul Mooney and Chris Booth (Ametek Gatan) for insightful discussions. The software used for this project was curated by SBGrid (Morin et al., 2013).

This work was supported by the National Institutes of Health, National Institute of General Medical Sciences (grant No. R35GM118099 to David A. Agard; grant No. R01HL134183 to David A. Agard; grant No. P50AI150476 to Yifan Cheng; grant No. S10OD020054 to UCSF CryoEM facility; grant No. S10OD021741 to UCSF Cryo-EM facility; grant No. S10OD026881 to UCSF Cryo-EM facility). Y.C. is an Investigator of Howard Hughes Medical Institute. A.F. is supported in part by a faculty scholar grant from the Howard Hughes medical institute and a Chan Zuckerberg Biohub investigator award.

\section{References}

Adams, P. D., Afonine, P. V., Bunkóczi, G., Chen, V. B., Davis, I. W., Echols, N., Headd, J. J., Hung, L. W., Kapral, G. J., Grosse-Kunstleve, R. W., McCoy, A. J., Moriarty, N. W., Oeffner, R., Read, R. J., Richardson, D. C., Richardson, J. S., Terwilliger, T. C. \& Zwart, P. H. (2010). Acta Crystallogr. Sect. D Biol. Crystallogr. 66, 213-221.

Asarnow, D., Palovcak, E., Cheng, Y. UCSF pyem v0.5. Zenodo https://doi.org/10.5281/zenodo.3576630 (2019)

Booth, C. R., Mooney, P. E., Lee, B. C., Lent, M. \& Gubbens, A. J. (2012). Microsc. Microanal. 18, 78-79.

Chen, S., McMullan, G., Faruqi, A. R., Murshudov, G. N., Short, J. M., Scheres,
S. H. W. \& Henderson, R. (2013). Ultramicroscopy. 135, 24-35. Emsley, P. \& Cowtan, K. (2004). Acta Crystallogr. Sect. D Biol. Crystallogr. 60, 2126-2132.

de la Rosa-Trevín, J. M., Quintana, A., del Cano, L., Zaldívar, A., Foche, I., Gutiérrez, J., Gómez-Blanco, J., Burguet-Castell, J., Cuenca-Alba, J., Abrishami, V., Vargas, J., Otón, J., Sharov, G., Vilas, J. L., Navas, J., Conesa, P., Kazemi, M., Marabini, R., Sorzano, C. O. S. \& Carazo, J. M. (2016). J. Struct. Biol.

Li, X., Mooney, P., Zheng, S., Booth, C. R., Braunfeld, M. B., Gubbens, S., Agard, D. A. \& Cheng, Y. (2013). Nat. Methods. 10, 584-590.

Li, X., Zheng, S. Q., Egami, K., Agard, D. A. \& Cheng, Y. (2013). J. Struct. Biol. 184, 251-260.

Mastronarde, D. N. (2005). J. Struct. Biol. 152, 36-51.

McMullan, G., Chen, S., Henderson, R. \& Faruqi, A. R. (2009). Ultramicroscopy. 109, 1126-1143.

McMullan, G., Faruqi, A. R. \& Henderson, R. (2016). Methods in Enzymology, Vol. 579, pp. 1-17.

Mooney, P. (2007). Methods Cell Biol. 2007, 661-719.

Morin, A., Eisenbraun, B., Key, J., Sanschagrin, P. C., Timony, M. A.,

Ottaviano, M. \& Sliz, P. (2013). Elife. 2013.

Nakane, T., Kotecha, A., Sente, A., McMullan, G., Masiulis, S., Brown, P. M. G.

E., Grigoras, I. T., Malinauskaite, L., Malinauskas, T., Miehling, J., Yu, L., Karia,

D., Pechnikova, E. V., Jong, E. de, Keizer, J., Bischoff, M., McCormack, J.,

Tiemeijer, P., Hardwick, S. W., Chirgadze, D. Y., Murshudov, G., Aricescu, A. R. \& Scheres, S. H. W. (2020). BioRxiv. 2020.05.22.110189.

Punjani, A., Rubinstein, J. L., Fleet, D. J. \& Brubaker, M. A. (2017). Nat. Methods. Rohou, A. \& Grigorieff, N. (2015). J. Struct. Biol. 192, 216-221.

Rosenthal, P. B. \& Henderson, R. (2003). J. Mol. Biol.

Scheres, S. H. W. (2012). J. Struct. Biol. 180, 519-530.

Suloway, C., Pulokas, J., Fellmann, D., Cheng, A., Guerra, F., Quispe, J., Stagg, S., Potter, C. S. \& Carragher, B. (2005). J. Struct. Biol. 151, 41-60.

Wu, M., Lander, G. C. \& Herzik, M. A. (2020). J. Struct. Biol. X. 4, 100020.

Yip, K. M., Fischer, N., Paknia, E., Chari, A. \& Stark, H. (2020). BioRxiv. 2020.05.21.106740.

Zhang, K., Pintilie, G. D., Li, S., Schmid, M. F. \& Chiu, W. (2020). BioRxiv.

Zheng, S. Q., Palovcak, E., Armache, J. P., Verba, K. A., Cheng, Y. \& Agard, D. A. (2017). Nat. Methods. 14, 331-332.

Zivanov, J., Nakane, T., Forsberg, B. O., Kimanius, D., Hagen, W. J. H., Lindahl, E. \& Scheres, S. H. W. (2018). Elife. 7,

Zivanov, J., Nakane, T. \& Scheres, S. H. W. (2019). IUCrJ. 6, 5-17. 
bioRxiv preprint doi: https://doi.org/10.1101/2020.11.08.372763; this version posted November 8, 2020. The copyright holder for this preprint (which was not certified by peer review) is the author/funder, who has granted bioRxiv a license to display the preprint in perpetuity. It is made available under aCC-BY 4.0 International license.

Sun et al., 08 Nov 2020 - preprint copy - BioRxiv

\section{Supplementary Information}

\begin{tabular}{|c|c|c|c|c|}
\hline \multicolumn{5}{|c|}{ K3 detector } \\
\hline Dose rate (counts/pix/s) & a & $b$ & $d$ & Average \\
\hline 3.57 & 1.003 & 0.0408 & 2.069 & \\
\hline 6.87 & 1.005 & 0.0836 & 2.201 & $a=1.0085 \pm 0.0058$ \\
\hline 12.76 & 1.01 & 0.1844 & 2.364 & \\
\hline 21.03 & 1.016 & 0.3368 & 2.346 & $d=2.245 \pm 0.138$ \\
\hline \multicolumn{5}{|c|}{ K2 detector (Li, Zheng et al., 2013) } \\
\hline Dose rate (counts/pix/s) & a & $\mathrm{b}$ & $d$ & Average \\
\hline 2.02 & 1.0037 & 0.0432 & 2.5812 & \\
\hline 4.05 & 1.0015 & 0.0991 & 2.5397 & $a=1.005 \pm 0.005$ \\
\hline 8.16 & 1.0001 & 0.2223 & 2.59 & \\
\hline 12.87 & 1.0035 & 0.3631 & 2.568 & $d=2.584 \pm 0.044$ \\
\hline 20.16 & 1.0168 & 0.5701 & 2.5398 & \\
\hline
\end{tabular}

Table S1. Summary of NPS curve fitting (Relevant to Figure S2). Least squares fitting of the NPS curves obtained from the K3 detector using the function: Intensity $=a-b{ }^{*}$ sinc2(frequency * $d$ ) (Li, Zheng et al., 2013) equation (1)). Results obtained previously from K2 are shown for comparison. The dampening effect of $\mathrm{b}$ reflects coincidence loss and is improved in the $\mathrm{K} 3$.

\begin{tabular}{|c|c|c|c|}
\hline \multicolumn{4}{|c|}{ NonCDS mode (1500 frames per second) } \\
\hline Dose rate (eps) & $\mathbf{k}=\mathbf{0}$ & 1 & 2 \\
\hline 1 & $99.69 \%$ & $0.31 \%$ & $0.00 \%$ \\
\hline 2 & $99.39 \%$ & $0.61 \%$ & $0.00 \%$ \\
\hline 4 & $98.78 \%$ & $1.21 \%$ & $0.01 \%$ \\
\hline 8 & $97.58 \%$ & $2.39 \%$ & $0.03 \%$ \\
\hline 16 & $95.21 \%$ & $4.67 \%$ & $0.11 \%$ \\
\hline 32 & $90.65 \%$ & $8.90 \%$ & $0.44 \%$ \\
\hline \multicolumn{4}{|c|}{ CDS mode (750 frames per second) } \\
\hline Dose rate (eps) & $k=0$ & 1 & 2 \\
\hline 1 & $99.39 \%$ & $0.61 \%$ & $0.00 \%$ \\
\hline 2 & $98.78 \%$ & $1.21 \%$ & $0.01 \%$ \\
\hline 4 & $97.58 \%$ & $2.39 \%$ & $0.03 \%$ \\
\hline 8 & $95.21 \%$ & $4.67 \%$ & $0.11 \%$ \\
\hline 16 & $90.65 \%$ & $8.90 \%$ & $0.44 \%$ \\
\hline 32 & $82.18 \%$ & $16.13 \%$ & $1.58 \%$ \\
\hline
\end{tabular}


bioRxiv preprint doi: https://doi.org/10.1101/2020.11.08.372763; this version posted November 8, 2020. The copyright holder for this preprint (which was not certified by peer review) is the author/funder, who has granted bioRxiv a license to display the preprint in perpetuity. It is made available under aCC-BY 4.0 International license.

Sun et al., DD MMM YYYY - preprint copy - BioRxiv

Table S2. Probability of number of electron events (k) overlapping for the K3 utilizing the effective internal frame rates with CDS mode off or on (Relevant to Table S1). Highlighted are two dose rates with the same probabilities of coincidence loss between the two modes. We used the binomial distribution model described previously (Li, Zheng et al., 2013) and the electron 'mask' d derived from fitting the intensities of NPS as the area to consider electron overlaps (Table S1).

\begin{tabular}{|c|c|c|c|c|c|}
\hline Magnification & Physical pixel size & $\begin{array}{c}\text { Dose rate } \\
\text { (e/pixel/sec) }\end{array}$ & No. of Particles & Resolution & $\begin{array}{c}\% \text { of Physical } \\
\text { Nyquist }\end{array}$ \\
\hline \multirow{4}{*}{105,000} & \multirow{4}{*}{$0.834 \AA ̊$ /pixel } & \multirow{4}{*}{4} & 10,000 & $2.05 \AA$ & $81 \%$ \\
\hline & & & 30,000 & $1.96 \AA$ & $85 \%$ \\
\hline & & & 60,000 & $1.89 \AA$ & $88 \%$ \\
\hline & & & 200,000 & $1.78 \AA$ & $94 \%$ \\
\hline \multirow{4}{*}{105,000} & \multirow{4}{*}{$0.834 \AA ̊$ /pixel } & \multirow{4}{*}{8} & 10,000 & $1.98 \AA$ & $84 \%$ \\
\hline & & & 30,000 & $1.91 \AA$ & $87 \%$ \\
\hline & & & 60,000 & $1.84 \AA$ & $91 \%$ \\
\hline & & & 200,000 & $1.75 \AA$ & $95 \%$ \\
\hline \multirow{4}{*}{105,000} & \multirow{4}{*}{$0.834 \AA ̊$ Apixel } & \multirow{4}{*}{16} & 10,000 & $2.16 \AA$ & $77 \%$ \\
\hline & & & 30,000 & $2.05 \AA$ & $81 \%$ \\
\hline & & & 60,000 & $1.94 \AA$ & $86 \%$ \\
\hline & & & 200,000 & $1.78 \AA$ & $94 \%$ \\
\hline \multirow{4}{*}{64,000} & \multirow{4}{*}{$1.346 \AA ̊$ A pixel } & \multirow{4}{*}{8} & 10,000 & $2.22 \AA$ & $121 \%$ \\
\hline & & & 30,000 & $2.04 \AA$ & $132 \%$ \\
\hline & & & 60,000 & $1.98 \AA$ & $136 \%$ \\
\hline & & & 200,000 & $1.91 \AA$ & $141 \%$ \\
\hline \multirow{4}{*}{64,000} & \multirow{4}{*}{$1.346 \AA ̊$ A pixel } & \multirow{4}{*}{16} & 10,000 & $2.32 \AA$ & $116 \%$ \\
\hline & & & 30,000 & $2.09 \AA$ & $129 \%$ \\
\hline & & & 60,000 & $2.00 \AA$ & $135 \%$ \\
\hline & & & 200,000 & $1.91 \AA$ & $141 \%$ \\
\hline
\end{tabular}

Table S3. Influence of dose rate on reconstructions of apoferritin from data recorded on the K3 detector (Relevant to Figure 1A). Results of 3D reconstructions from apoferritin data sets collected at different dose rates. All resolutions are reported in absolute angstrom and percentage of physical Nyquist frequency calculated from gold standard FSC estimates with soft mask. 
bioRxiv preprint doi: https://doi org/10.1101/2020.11.08.372763; this version posted November 8,2020 . The copyright holder for this preprint (which was not certified by peer review) is the author/funder, who has granted bioRxiv a license to display the preprint in perpetuity. It is made available under aCC-BY 4.0 International license.

Sun et al., 08 Nov 2020 - preprint copy - BioRxiv

\begin{tabular}{|c|c|c|c|c|c|c|}
\hline Magnification & Physical pixel size & $\begin{array}{c}\text { Dose rate } \\
\text { (e/pixel/sec) }\end{array}$ & Mode & No. of Particles & Resolution & $\begin{array}{c}\% \text { of Physical } \\
\text { Nyquist }\end{array}$ \\
\hline \multirow{4}{*}{64,000} & \multirow{4}{*}{$1.346 \AA ̊ /$ pixel } & \multirow{4}{*}{8} & CDS & 10,000 & $2.22 \AA$ & $121 \%$ \\
\hline & & & & 30,000 & $2.04 \AA$ & $132 \%$ \\
\hline & & & & 60,000 & $1.98 \AA$ & $136 \%$ \\
\hline & & & & 200,000 & $1.91 \AA$ & $141 \%$ \\
\hline \multirow{4}{*}{64,000} & \multirow{4}{*}{$1.346 \AA ̊ ̊ n / p i x e l$} & \multirow{4}{*}{16} & NonCDS & 10,000 & $2.32 \AA$ & $116 \%$ \\
\hline & & & & 30,000 & $2.23 \AA$ & $121 \%$ \\
\hline & & & & 60,000 & $2.11 \AA$ & $128 \%$ \\
\hline & & & & 200,000 & $1.98 \AA$ & $136 \%$ \\
\hline \multirow{4}{*}{105,000} & \multirow{4}{*}{$0.834 \AA ̊$ Apixel } & \multirow{4}{*}{4} & CDS & 10,000 & $2.05 \AA$ & $81 \%$ \\
\hline & & & & 30,000 & $1.96 \AA$ & $85 \%$ \\
\hline & & & & 60,000 & $1.89 \AA$ & $88 \%$ \\
\hline & & & & 200,000 & $1.78 \AA$ & $94 \%$ \\
\hline \multirow{4}{*}{105,000} & \multirow{4}{*}{$0.834 \AA ̊ /$ pixel } & \multirow{4}{*}{8} & NonCDS & 10,000 & $2.16 \AA$ & $77 \%$ \\
\hline & & & & 30,000 & $2.03 \AA$ & $82 \%$ \\
\hline & & & & 60,000 & $1.98 \AA$ & $84 \%$ \\
\hline & & & & 200,000 & $1.91 \AA$ & $89 \%$ \\
\hline
\end{tabular}

Table S4. Benefits of imaging apoferritin in CDS mode when comparing data sets with equivalent coincidence loss of electron events (Relevant to Figure 2). Results of $3 \mathrm{D}$ reconstructions from apoferritin data sets collected at constant coincidence loss. All resolutions are reported in absolute angstrom and percentage of physical Nyquist frequency calculated from gold standard FSC estimates with soft masks. 
bioRxiv preprint doi: https://doi. org/10.1101/2020 11.08.372763; this version posted November 8,2020 . The copyright holder for this preprint (which was not certified by peer review) is the author/funder, who has granted bioRxiv a license to display the preprint in perpetuity. It is made available under aCC-BY 4.0 International license.

Sun et al., DD MMM YYYY - preprint copy - BioRxiv

\begin{tabular}{|c|c|c|c|c|c|}
\hline $\begin{array}{c}\text { Magnification/ } \\
\text { Physical pixel size }\end{array}$ & $\begin{array}{c}\text { Dose rate } \\
\text { (e/pixel/sec) }\end{array}$ & Mode & No. of Particles & $\begin{array}{l}\text { Resolution }(\AA \AA) \\
\text { (soft mask) }\end{array}$ & $\begin{array}{c}\% \text { of Physical } \\
\text { Nyquist }\end{array}$ \\
\hline \multirow{4}{*}{$\begin{array}{c}64,000 \\
(1.346 \AA \text { A }\end{array}$} & 8 & CDS & 10,000 & 2.22 & $121 \%$ \\
\hline & & & 30,000 & 2.04 & $132 \%$ \\
\hline & & & 60,000 & 1.98 & $136 \%$ \\
\hline & & & 200,000 & 1.91 & $141 \%$ \\
\hline \multirow{4}{*}{$\begin{array}{c}64,000 \\
(1.346 \AA \text { A /pixel })\end{array}$} & 8 & NonCDS & 10,000 & 2.28 & $118 \%$ \\
\hline & & & 30,000 & 2.07 & $130 \%$ \\
\hline & & & 60,000 & 2.01 & $134 \%$ \\
\hline & & & 200,000 & 1.94 & $139 \%$ \\
\hline \multirow{4}{*}{$\begin{array}{c}105,000 \\
(0.834 \AA / \text { pixel })\end{array}$} & 8 & CDS & 10,000 & 1.98 & $84 \%$ \\
\hline & & & 30,000 & 1.91 & $87 \%$ \\
\hline & & & 60,000 & 1.84 & $91 \%$ \\
\hline & & & 200,000 & 1.75 & $95 \%$ \\
\hline \multirow{4}{*}{$\begin{array}{c}105,000 \\
(0.834 \AA / \text { pixel })\end{array}$} & 8 & NonCDS & 10,000 & 2.16 & $77 \%$ \\
\hline & & & 30,000 & 2.03 & $82 \%$ \\
\hline & & & 60,000 & 1.98 & $84 \%$ \\
\hline & & & 200,000 & 1.94 & $85 \%$ \\
\hline
\end{tabular}

Table S5. Practical consideration of imaging apoferritin using CDS mode at the recommended dose rate of 8eps (Relevant to Figure 3). Results of $3 \mathrm{D}$ reconstructions from apoferritin data sets collected at constant dose rate. All resolutions are reported in absolute angstrom and percentage of physical Nyquist frequency calculated from gold standard FSC estimates (Chen et al., 2013; Rosenthal \& Henderson, 2003). 
bioRxiv preprint doi: https://doi org/10.1101/202011.08.372763; this version posted November 8,2020 . The copyright holder for this preprint (which was not certified by peer review) is the author/funder, who has granted bioRxiv a license to display the preprint in perpetuity. It is made available under aCC-BY 4.0 International license.

Sun et al., 08 Nov 2020 - preprint copy - BioRxiv

\begin{tabular}{|c|c|}
\hline \multicolumn{2}{|c|}{ Apoferritin (8eps, 105kx, CDS) } \\
\hline Data collection and processing & \\
\hline Magnification & 105,000 \\
\hline Voltage (kV) & 300 \\
\hline Electron exposure $\left(\mathrm{e}-/ \AA^{2}\right)$ & 69 \\
\hline Defocus range $(\mu \mathrm{m})$ & -0.3 to -0.7 \\
\hline Pixel size $(\AA ̊)$ & $\begin{array}{c}0.834 \text { (physical pixel size) } \\
0.417 \text { (super) }\end{array}$ \\
\hline Symmetry imposed & $\mathrm{O}$ \\
\hline Map resolution $(\AA)$ & 1.65 (FSC 0.143) \\
\hline \multicolumn{2}{|l|}{ Refinement } \\
\hline Initial model used (PDB code) & 5ОВA/5OBB \\
\hline $\begin{array}{c}\text { Model resolution }(\AA) \\
\text { (FSC threshold } 0.143 / 0.5)\end{array}$ & $1.5 / 1.7(0.143 / 0.5)$ \\
\hline $\begin{array}{c}\text { Model composition } \\
\text { Atoms non- } \mathrm{H} \text { and } \mathrm{H} \\
\text { Protein residues } \\
\text { Water }\end{array}$ & $\begin{array}{c}68186(\mathrm{H}: 32643) \\
4128 \\
1727\end{array}$ \\
\hline $\begin{array}{c}B \text { factors }\left(\AA^{2}\right) \min / \text { max } / \text { mean } \\
\text { Protein } \\
\text { Water }\end{array}$ & $\begin{array}{l}5 / 101 / 21 \\
10 / 66 / 22\end{array}$ \\
\hline $\begin{array}{c}\text { R.m.s. deviations } \\
\text { Bond lengths }(\AA)(\#>4 \sigma) \\
\text { Bond angles }\left(\left(^{\circ}\right)(\#>4 \sigma)\right.\end{array}$ & $\begin{array}{l}0.001(0) \\
0.342(0)\end{array}$ \\
\hline $\begin{array}{c}\text { Validation } \\
\text { MolProbity score } \\
\text { Clashscore } \\
\text { Poor rotamers (\%) }\end{array}$ & $\begin{array}{l}0.75 \\
0.78 \\
0.05\end{array}$ \\
\hline $\begin{array}{l}\text { Ramachandran plot } \\
\text { Favored (\%) } \\
\text { Allowed (\%) } \\
\text { Disallowed (\%) }\end{array}$ & $\begin{array}{c}98.87 \\
1.13 \\
0.00\end{array}$ \\
\hline
\end{tabular}

Table S6. Statistics of data collection, 3D refinement, and atomic model for data collected at 8eps and 105kx in CDS mode (Relevant to Figure 5). The model validation was carried out in Phenix software (Adams et al., 2010), using medium sharpened cryo-EM full density map. 
bioRxiv preprint doi: https://doi.org/10.1101/2020.11.08.372763; this version posted November 8. 2020. The copvriaht holder for this preprint (which was not certified by peer review) is the author/funder, who has granted bioRxiv a license to display the preprint in perpetuity. It is made available under aCC-BY 4.0 International license.

Sun et al., DD MMM YYYY - preprint copy - BioRxiv

\begin{tabular}{|c|c|c|c|c|}
\hline \multicolumn{4}{|c|}{ Aldolase 64kx 8eps CDS } \\
\hline \multirow{2}{*}{ \# of particles } & \multicolumn{2}{|c|}{ Auto 3D Refinement } & Bayesian Polishing \\
\hline 252000 & (no mask) & $2.66 \AA / 99 \%$ & (no mask) & (soft mask) \\
\hline 200000 & $3.01 \AA$ & $2.88 \AA$ & $2.55 \AA / 105 \%$ \\
\hline & & $2.73 \AA / 99 \%$ & $2.97 \AA$ & $2.66 \AA 101 \%$ \\
\hline 252000 & $3.11 \AA$ & Aldolase $64 \mathrm{kx} 8 \mathrm{eps}$ nonCDS & $2.92 \mathrm{~A} \AA \mathrm{A} / 107 \%$ \\
\hline 200000 & $3.15 \AA$ & $2.69 \AA / 100 \%$ & $2.97 \AA$ & $2.62 \AA / 103 \%$ \\
\hline
\end{tabular}

\begin{tabular}{|c|c|c|c|c|}
\hline \multicolumn{5}{|c|}{ Apoferritin 64kx 8eps CDS } \\
\hline & \multicolumn{2}{|c|}{ Auto 3D Refinement } & Bayesian Polishing \\
\hline \# of particles & (no mask) & (soft mask) & $2.01 \AA$ & $($ soft mask) \\
\hline 373000 & $2.04 \AA$ & $1.86 \AA / 144 \%$ & $2.06 \AA$ & $1.80 \AA / 149 \%$ \\
\hline 200000 & $2.11 \AA$ & $1.91 \AA / 141 \%$ & $1.87 \AA / 144 \%$ \\
\hline 256000 & $2.10 \AA$ & Apoferritin $64 \mathrm{kx} 8 \mathrm{eps}$ nonCDS & \\
\hline 200000 & $2.10 \AA$ & $1.89 \AA / 142 \%$ & $2.02 \AA$ & $1.87 \AA / 144 \%$ \\
\hline
\end{tabular}

\begin{tabular}{|c|c|c|c|c|}
\hline \multicolumn{5}{|c|}{ Apoferritin 105kx 8eps CDS } \\
\hline & \multicolumn{2}{|c|}{ Auto 3D Refinement } & \multicolumn{2}{c|}{ Bayesian Polishing } \\
\hline \# of particles & (no mask) & (soft mask) & $1.76 \AA$ & (soft mask) \\
\hline 571000 & $1.81 \AA$ & $1.71 \AA / 95 \%$ & $1.79 \AA$ & $1.65 \AA / 101 \%$ \\
\hline 200000 & $1.88 \AA$ & $1.75 \AA / 95 \%$ & \\
\hline & & Apoferritin $105 \mathrm{kx} 8 \mathrm{eps}$ nonCDS & \\
\hline & $1.97 \AA$ & $1.86 \AA$ & $1.90 \AA$ & $1.76 \AA / 95 \%$ \\
\hline 241000 & $2.01 \AA$ & $1.87 \AA$ & $1.98 \AA$ & $1.86 / 90 \%$ \\
\hline
\end{tabular}

Table S7. Resolution improvements from per-particle motion correction (Relevant to Figure S4). Results of 3D reconstructions from apoferritin and aldolase data sets before and after Bayesian polishing, with and without masking. All resolutions are reported in absolute angstrom and percentage of physical Nyquist frequency calculated from gold standard FSC estimates. All resolutions are reported in absolute angstrom and percentage of physical Nyquist frequency calculated from gold standard FSC estimates (Chen et al., 2013; Rosenthal \& Henderson, 2003). 
bioRxiv preprint doi: https://doi org/10.1101/202011.08.372763; this version posted November 8,2020 . The copyright holder for this preprint (which was not certified by peer review) is the author/funder, who has granted bioRxiv a license to display the preprint in perpetuity. It is made available under aCC-BY 4.0 International license.

Sun et al., 08 Nov 2020 - preprint copy - BioRxiv

\begin{tabular}{|c|c|c|c|c|c|c|}
\hline Magnification & $\begin{array}{c}\text { Physical pixel } \\
\text { size }\end{array}$ & $\begin{array}{c}\text { Dose rate } \\
\text { (e/pixel/sec) }\end{array}$ & Mode & No. of Particles & Resolution & $\begin{array}{c}\% \text { of Physical } \\
\text { Nyquist }\end{array}$ \\
\hline \multirow{4}{*}{64,000} & \multirow{4}{*}{$\begin{array}{l}1.346 \\
\AA \text { A } / \text { pixel }\end{array}$} & 8 & CDS & 10,000 & $3.5 \AA$ & $77 \%$ \\
\hline & & & & 30,000 & $3.2 \AA$ & $85 \%$ \\
\hline & & & & 60,000 & $2.9 \AA$ & $92 \%$ \\
\hline & & & & 200,000 & $2.7 \AA$ & $100 \%$ \\
\hline \multirow{4}{*}{64,000} & \multirow{4}{*}{$\begin{array}{l}1.346 \\
\AA \text { A } / \text { pixel }\end{array}$} & 8 & NonCDS & 10,000 & $4.0 \AA$ & $67 \%$ \\
\hline & & & & 30,000 & $3.3 \AA$ & $83 \%$ \\
\hline & & & & 60,000 & $3.2 \AA$ & $84 \%$ \\
\hline & & & & 200,000 & $2.8 \AA$ & $95 \%$ \\
\hline \multirow{4}{*}{64,000} & \multirow{4}{*}{$\begin{array}{c}1.346 \\
\AA \text { \& } / \text { pixel }\end{array}$} & 16 & CDS & 10,000 & $3.8 \AA$ & $71 \%$ \\
\hline & & & & 30,000 & $3.3 \AA$ & $83 \%$ \\
\hline & & & & 60,000 & $3.0 \AA$ & $89 \%$ \\
\hline & & & & 200,000 & $2.7 \AA$ & $99 \%$ \\
\hline \multirow{4}{*}{64,000} & \multirow{4}{*}{$\begin{array}{l}1.346 \\
\AA \text { A } / \text { pixel }\end{array}$} & 16 & NonCDS & 10,000 & $3.4 \AA$ & $79 \%$ \\
\hline & & & & 30,000 & $3.1 \AA$ & $87 \%$ \\
\hline & & & & 60,000 & $2.9 \AA$ & $92 \%$ \\
\hline & & & & 200,000 & $2.7 \AA$ & $99 \%$ \\
\hline
\end{tabular}

Table S8. Balancing the effects of dose rate, coincidence loss, and noise reduction when imaging in CDS and nonCDS mode for aldolase (Relevant to Figure S6). Comparison of all aldolase data sets collected at 64,000x magnification. All resolutions are reported in absolute angstrom and percentage of physical Nyquist frequency calculated from gold standard FSC estimates (Chen et al., 2013; Rosenthal \& Henderson, 2003). 
bioRxiv preprint doi: https://doi.org/10.1101/2020.11.08.372763; this version posted November 8, 2020. The copyright holder for this preprint (which was not certified by peer review) is the author/funder, who has granted bioRxiv a license to display the preprint in perpetuity. It is made available under aCC-BY 4.0 International license.

Sun et al., DD MMM YYYY - preprint copy - BioRxiv

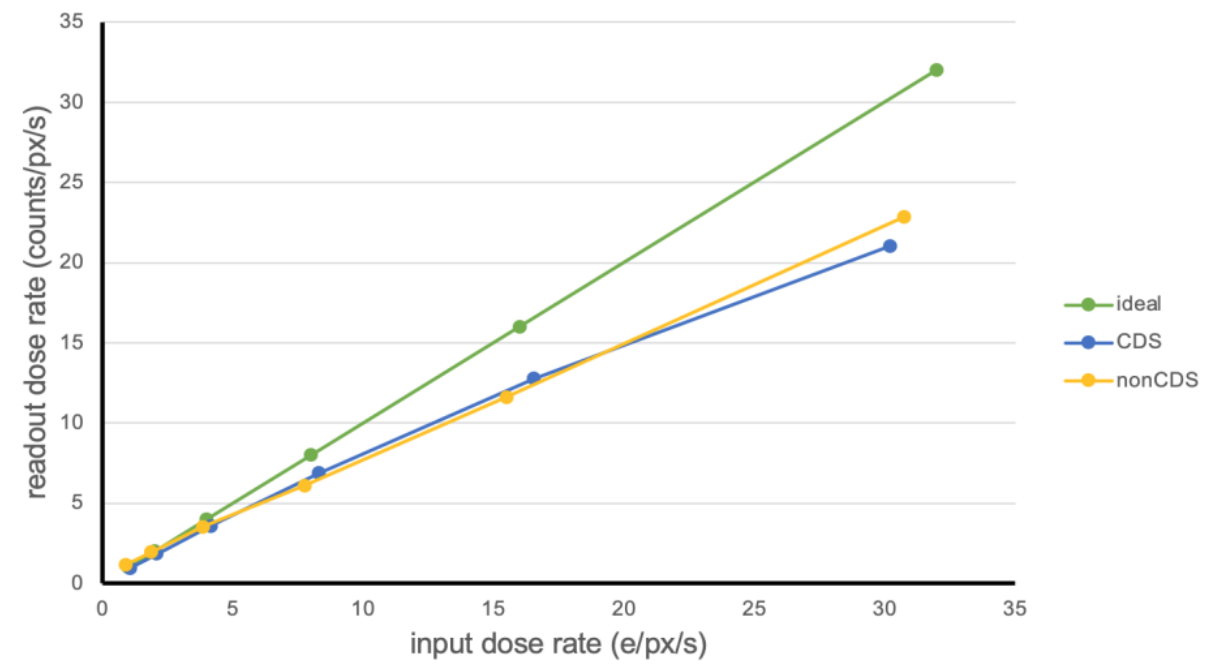

Figure S1. Coincidence loss for the Gatan K3 detector. Detector counts converted to electron dose of the Gatan K3 operating in counted mode with and without CDS mode compared to 1 to 1 conversion. Electron counts can be separated as independent events at lower dose rates which fall off as the incident electron dose rate increases.

(A) $3.57 \mathrm{counts} / \mathrm{pixel} / \mathrm{sec} \quad 6.87 \mathrm{counts} / \mathrm{pixel} / \mathrm{sec} 12.76 \mathrm{counts} / \mathrm{pixel} / \mathrm{sec} 21.02 \mathrm{counts} / \mathrm{pixel} / \mathrm{sec}$
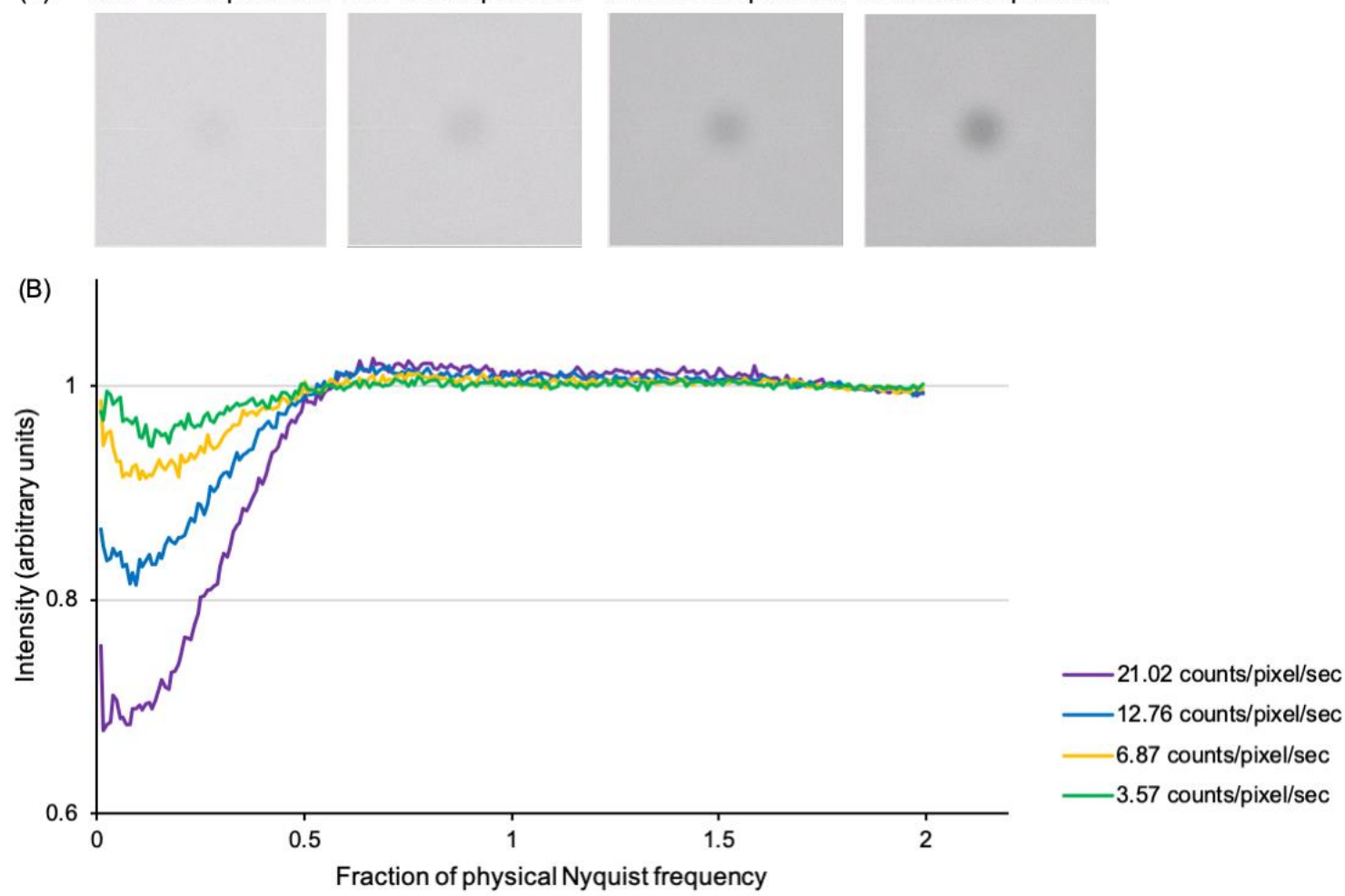

Figure S2. Noise power spectra (NPS) acquired at various dose rates. (A) Fourier transforms of empty super-resolution counting images recorded with different dose rates in CDS mode. (B) Simulated noise power spectra at different dose rates, by using the normalized sinc function (Li, Zheng et al., 2013) equation (1). The dip of the NPS curve at low frequencies describes coincidence loss with increasing dose rate. 
bioRxiv preprint doi: https://doi.org/10.1101/2020.11.08.372763; this version posted November 8, 2020. The copyright holder for this preprint (which was not certified by peer review) is the author/funder, who has granted bioRxiv a license to display the preprint in perpetuity. It is made available under aCC-BY 4.0 International license.

Sun et al., 08 Nov 2020 - preprint copy - BioRxiv

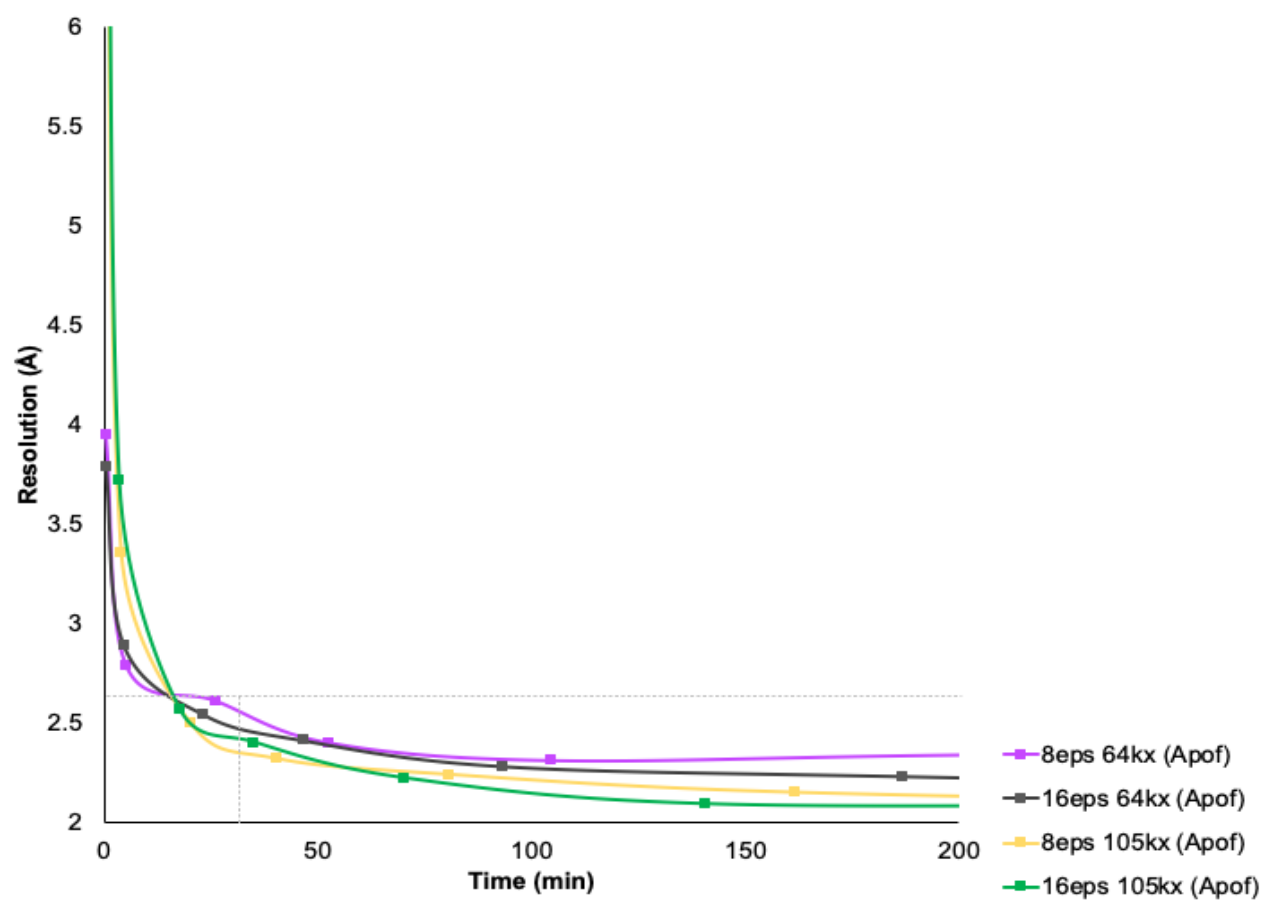

Figure S3. Time versus resolution plot for apoferritin refined with $\mathbf{C} 1$ symmetry. All particles from the same selected micrographs in Figure 4 were processed in cryoSPARC v2.12.4 (Punjani et al., 2017), following standard protocols without 3D classification as in Figure 4 with C1 symmetry. The 8eps $105 \mathrm{kx}$ CDS mode data reconstructed below 2 Å resolution after $\sim 8$ hours of data.

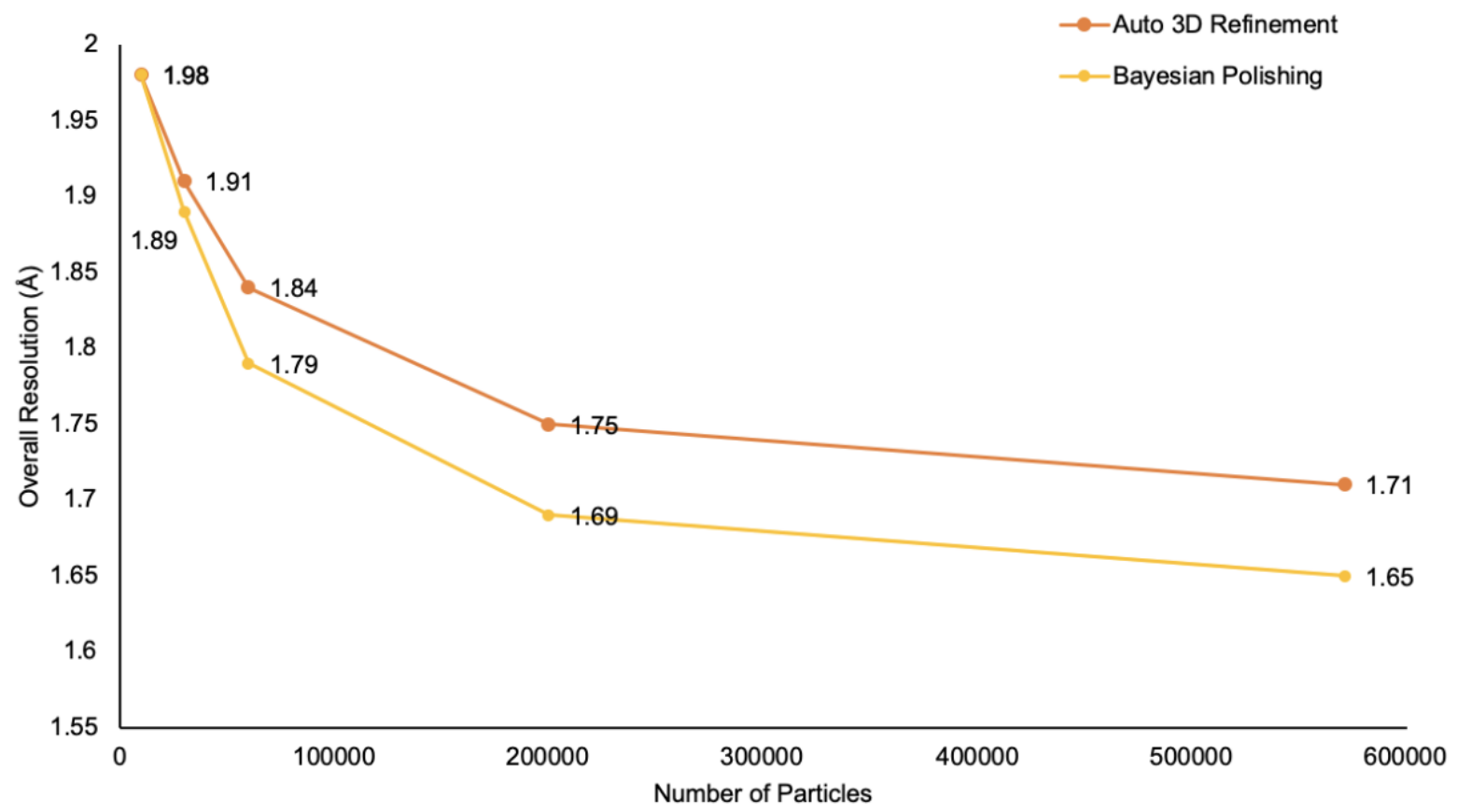

Figure S4. Resolution improvements from per-particle motion correction. To further improve the resolution of the apoferritin data sets collected using 9-hole image shift, Bayesian polishing was performed to further optimize per-particle beam-induced motion tracks, followed by another round of autorefinement (Zivanov et al., 2019). All resolutions are reported in absolute angstrom and percentage of physical Nyquist frequency calculated from gold standard FSC estimates (Chen et al., 2013; Rosenthal \& Henderson, 2003). Results are plotted as a function of percent of physical Nyquist frequency versus particle number and achievable resolutions are labelled. 
bioRxiv preprint doi: https://doi.org/10.1101/2020.11.08.372763; this version posted November 8, 2020. The copyright holder for this preprint (which was not certified by peer review) is the author/funder, who has granted bioRxiv a license to display the preprint in perpetuity. It is made available under aCC-BY 4.0 International license.

Sun et al., DD MMM YYYY - preprint copy - BioRxiv

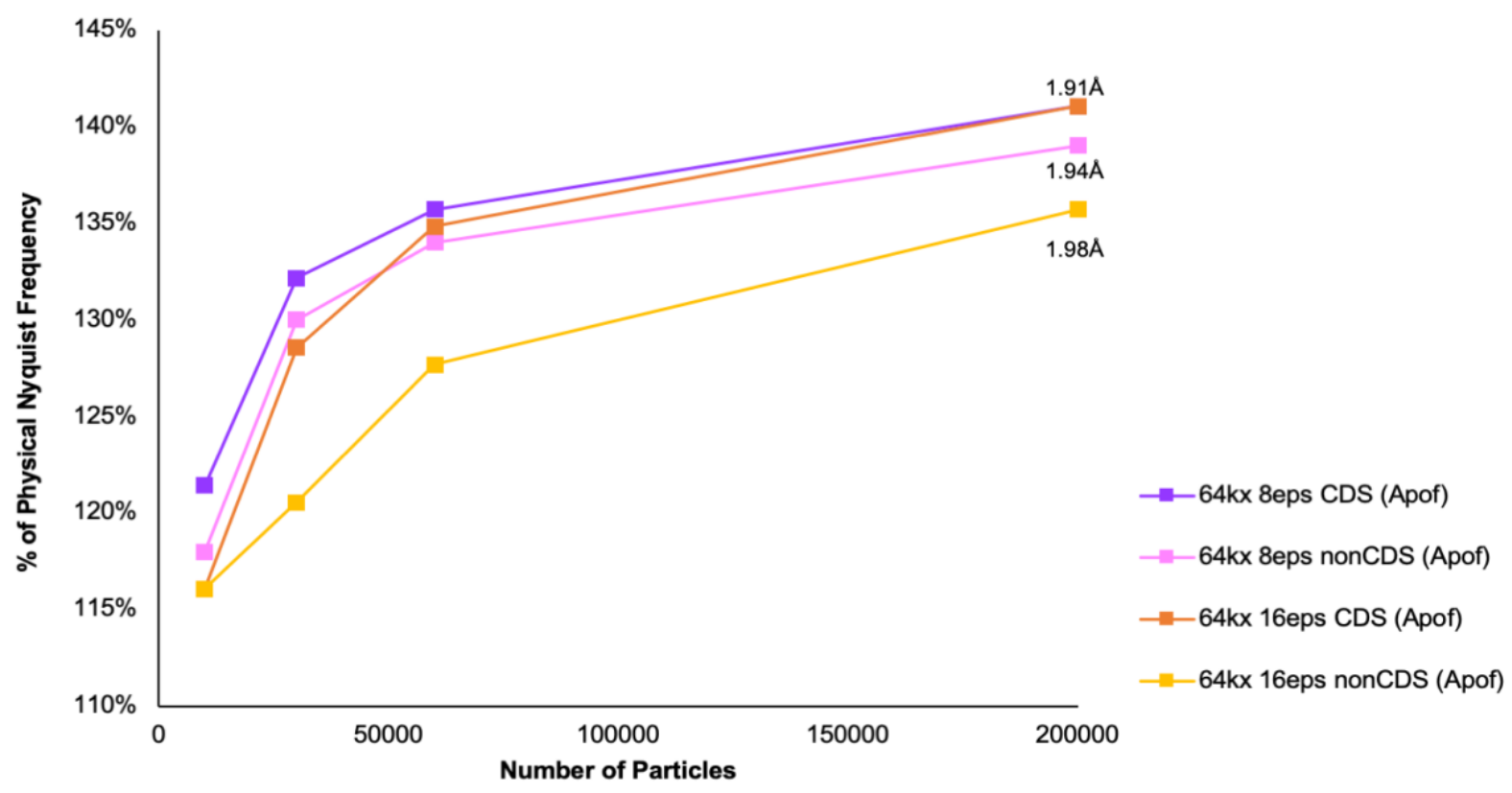

Figure S5. Balancing the effects of dose rate, coincidence loss, and noise reduction when imaging in CDS and nonCDS mode for apoferritin. Comparison of apoferritin data sets collected at 64,000x magnification with different dose rates. Results are plotted as a function of percent of physical Nyquist frequency versus particle number and highest achievable resolutions are labelled. All resolutions are reported in absolute angstrom and percentage of physical Nyquist frequency calculated from gold standard FSC estimates (Chen et al., 2013; Rosenthal \& Henderson, 2003).

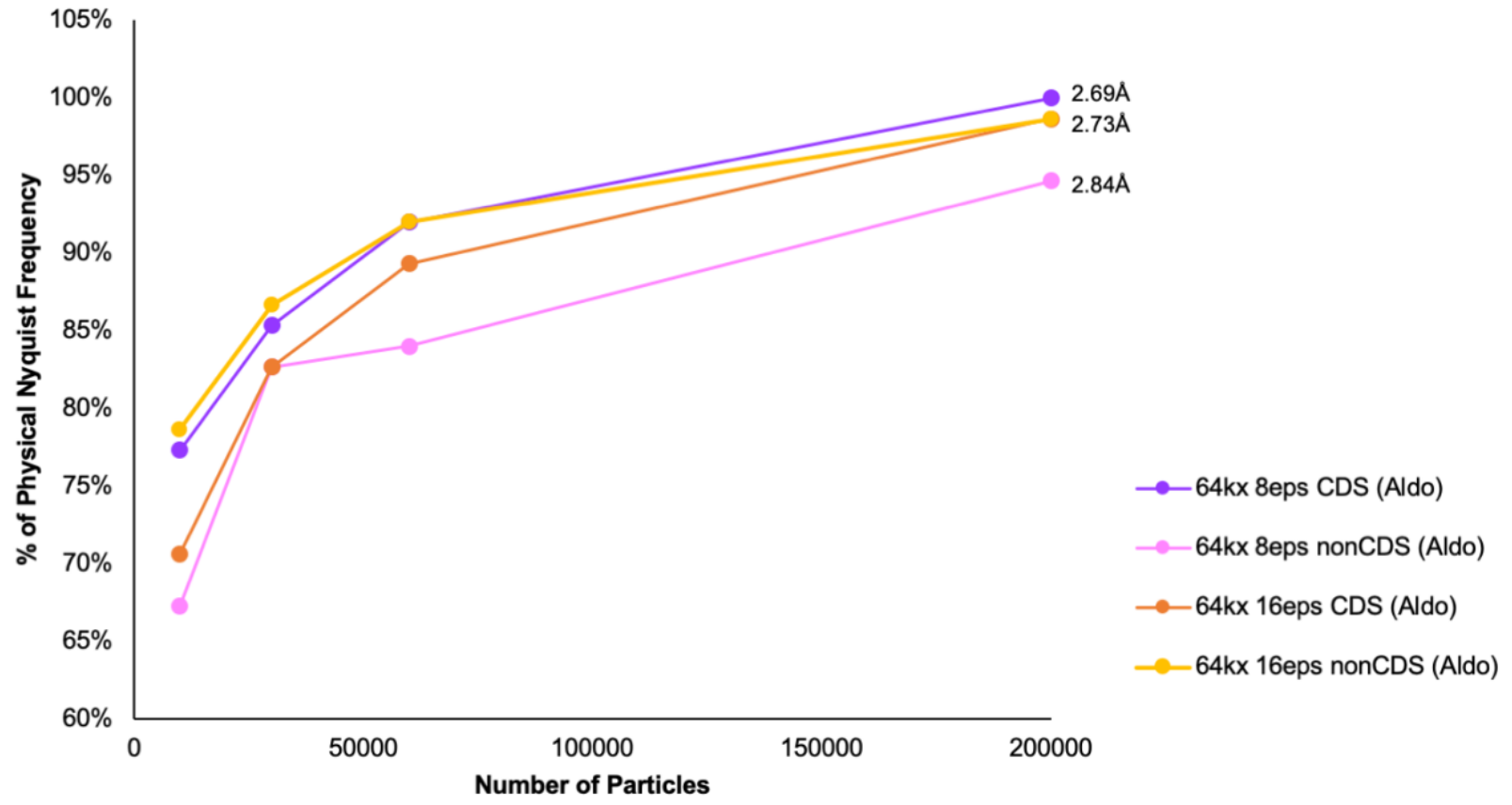

Figure S6. Balancing the effects of dose rate, coincidence loss, and noise reduction when imaging in CDS and nonCDS mode for aldolase. Comparison of aldolase data sets collected at 64,000x magnification. These results are plotted as a function of percent of physical Nyquist frequency versus particle number and highest achievable resolutions are labelled. All resolutions are reported in absolute angstrom and percentage of physical Nyquist frequency calculated from gold standard FSC estimates (Chen et al., 2013; Rosenthal \& Henderson, 2003). 
bioRxiv preprint doi: https://doi org/10.1101/2020.11.08.372763. this version posted November 8,2020 . The copyright holder for this preprint (which was not certified by peer review) is the author/funder, who has granted bioRxiv a license to display the preprint in perpetuity. It is made available under aCC-BY 4.0 International license.

Sun et al., 08 Nov 2020 - preprint copy - BioRxiv
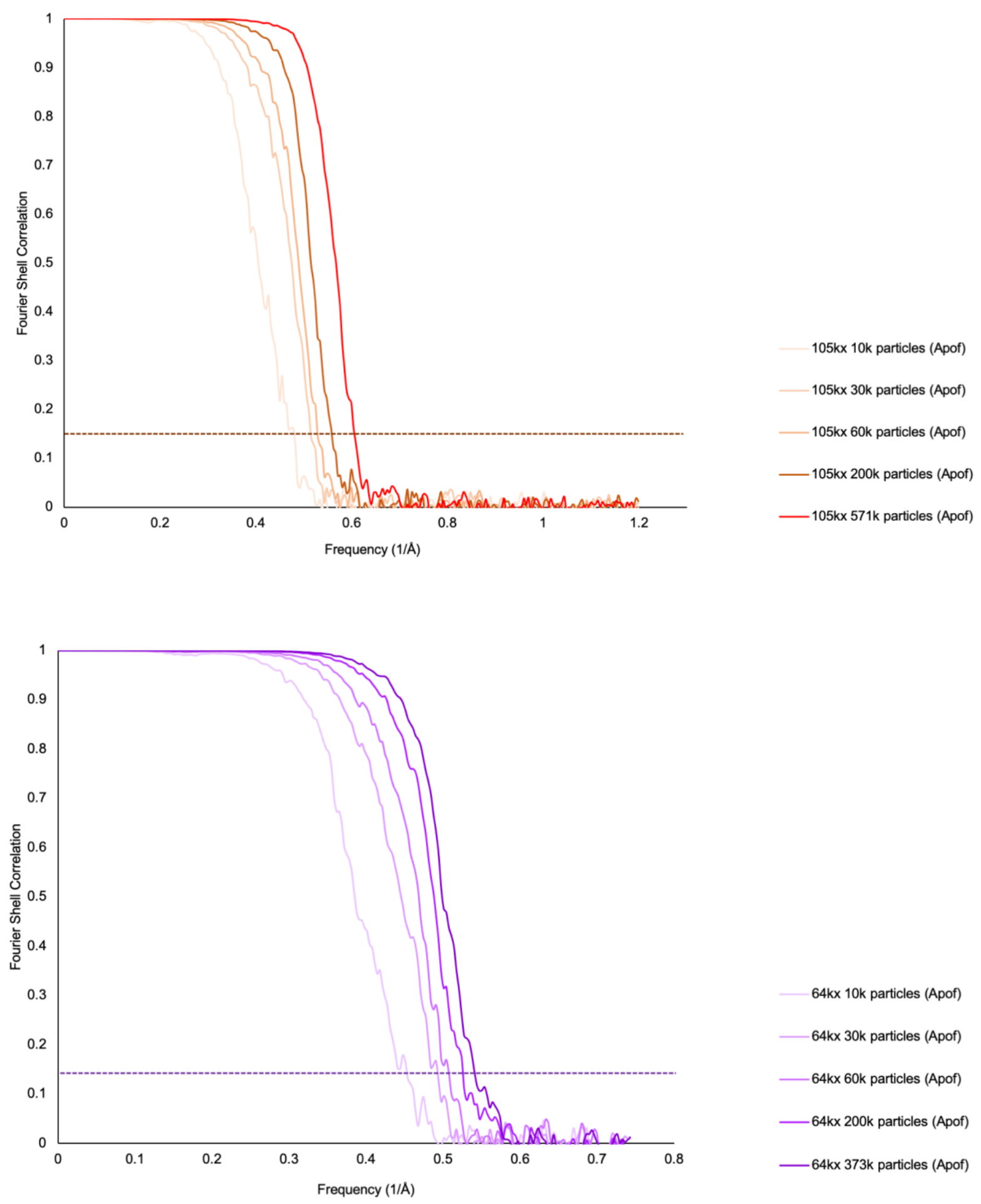

Figure S7. Gold-standard FSC curves for the apoferritin reconstructions from data subsets collected at 105kx (upper panel) and 64kx (lower panel). 